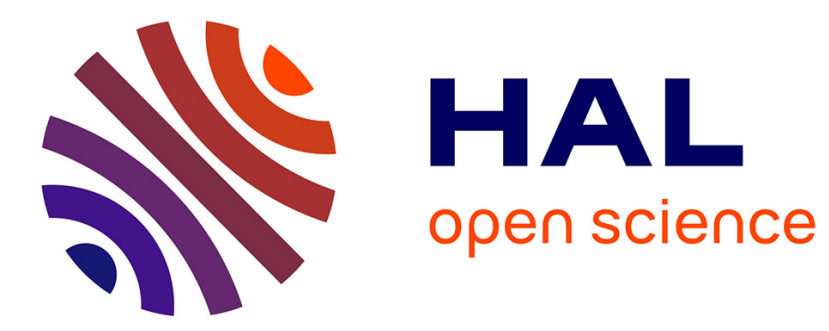

\title{
Global Singularity-Free Aerodynamic Model for Algorithmic Flight Control of Tail Sitters
}

Leandro Ribeiro Lustosa, François Defaÿ, Jean-Marc Moschetta

\section{To cite this version:}

Leandro Ribeiro Lustosa, François Defaÿ, Jean-Marc Moschetta. Global Singularity-Free Aerodynamic Model for Algorithmic Flight Control of Tail Sitters. Journal of Guidance, Control, and Dynamics, 2019, 42 (2), pp.303-316. 10.2514/1.G003374 . hal-03035938

\section{HAL Id: hal-03035938 \\ https://hal.science/hal-03035938}

Submitted on 2 Dec 2020

HAL is a multi-disciplinary open access archive for the deposit and dissemination of scientific research documents, whether they are published or not. The documents may come from teaching and research institutions in France or abroad, or from public or private research centers.
L'archive ouverte pluridisciplinaire HAL, est destinée au dépôt et à la diffusion de documents scientifiques de niveau recherche, publiés ou non, émanant des établissements d'enseignement et de recherche français ou étrangers, des laboratoires publics ou privés. 


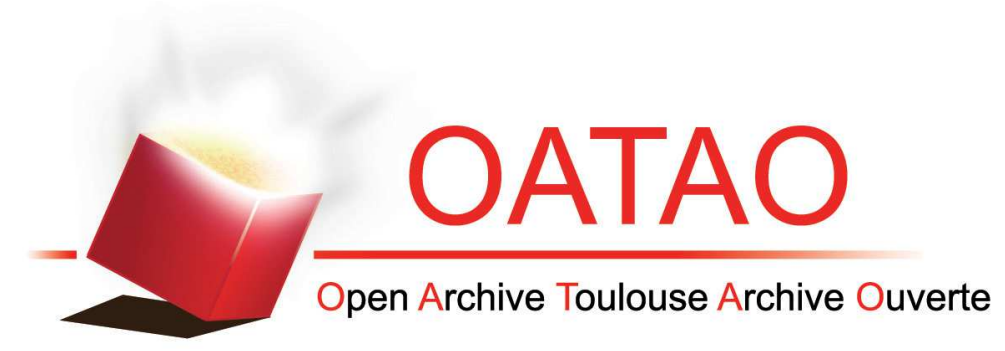

\section{Open Archive Toulouse Archive Ouverte (OATAO)}

OATAO is an open access repository that collects the work of some Toulouse researchers and makes it freely available over the web where possible.

This is an author's version published in: https://oatao.univ-toulouse.fr/21708

Official URL: https://doi.org/10.2514/1.G003374

\section{To cite this version :}

Lustosa, Leandro Ribeiro and Defaÿ, François and Moschetta, Jean-Marc Global Singularity-Free Aerodynamic Model for Algorithmic Flight Control of Tail Sitters. (2019) Journal of Guidance, Control, and Dynamics, 42 (2). 303-316. ISSN 0731-5090

Any correspondence concerning this service should be sent to the repository administrator: tech-oatao@listes-diff.inp-toulouse.fr 


\title{
Global Singularity-Free Aerodynamic Model for Algorithmic Flight Control of Tail Sitters
}

\author{
Leandro R. Lustosa, $\stackrel{*}{*}$ François Defaÿ, $\doteq$ and Jean-Marc Moschetta \\ ISAE-SUPAERO, Université de Toulouse, 31400 Toulouse, France
}

DOI: $10.2514 / 1 . G 003374$

\begin{abstract}
This paper addresses fundamental issues in tail-sitting and transition flight aerodynamics modeling in view of sumof-squares (SOS) algorithmic guidance and control design. A novel approach, called $\phi$ theory, for modeling aerodynamic forces and moments is introduced herein. It yields polynomial-like differential equations of motion that are well suited to SOS solvers for real-time algorithmic guidance and control law synthesis. The proposed $\phi$ theory allows for first principles model parameter identification and captures dominant dynamical features over the entire flight envelope. Furthermore, $\phi$ theory yields numerically stable and consistent models for $\mathbf{3 6 0}$ deg angles of attack and sideslip. Additionally, an algorithm is provided for analytically computing all feasible longitudinal flight operating points. Finally, to establish $\phi$-theory validity, predicted trim points and wind-tunnel experiments are compared.
\end{abstract}

$\mathcal{B}$

$B \in \mathbb{R}^{3 \times 3}$

$b \in \mathbb{R}_{+}^{*}$

$C \in \mathbb{R}^{3}$

$C \in \mathbb{R}^{6 \times 6}$

$C_{D} \in \mathbb{R}$

$C_{L} \in \mathbb{R}$

$c \in \mathbb{R}_{+}^{*}$

$D_{b}^{a} \in \mathrm{SO}(3)$

$\boldsymbol{F}^{\prime} \in \mathbb{R}^{3}$

$g \in \mathbb{R}^{3}$

$J \in \mathbb{R}^{3 \times 3}$

$J_{p} \in \mathbb{R}^{3 \times 3}$

$\boldsymbol{K} \in \mathbb{R}^{3}$

$k_{f} \in \mathbb{R}$

$k_{m} \in \mathbb{R}$

$M \in \mathbb{R}^{*}$

$\boldsymbol{M}^{\prime} \in \mathbb{R}^{3}$

$m \in \mathbb{R}_{+}^{*}$

$R e \in \mathbb{R}_{+}^{*}$

$\boldsymbol{r}^{A / B} \in \mathbb{R}^{3}$

$S \in \mathbb{R}_{+}^{*}$

$S_{++}^{N}$

$\boldsymbol{T} \in \mathbb{R}^{3}$

$\boldsymbol{T}_{1} \in \mathbb{R}^{3}$

$\boldsymbol{T}_{2} \in \mathbb{R}^{3}$

$\boldsymbol{v} \in \mathbb{R}^{3}$

$\boldsymbol{v}_{\infty} \in \mathbb{R}^{3}$

$\alpha \in \mathbb{R}$

$\beta \in \mathbb{R}$

$\delta_{1} \in \mathbb{R}$

$\delta_{2} \in \mathbb{R}$

$\zeta_{f} \in \mathbb{R}^{3}$

$\boldsymbol{\eta} \in \mathbb{R}^{6}$

$\rho \in \mathbb{R}_{+}^{*}$

$\sigma_{i}(\cdot) \in \mathbb{R}$

\section{Nomenclature}

$=$ body frame

$=$ reference dimensions matrix

$=$ reference wingspan

$=$ Buckingham- $\pi$-based force coefficients

$=$ wing screw reference matrix

$=$ Buckingham- $\pi$-based drag coefficient

$=$ Buckingham- $\pi$-based lift coefficient

$=$ reference chord

$=$ direction cosine matrix from frame $A$ to frame $B$

$=$ Buckingham- $\pi$-based forces

$=$ gravity

$=$ inertia matrix

$=$ propeller inertia matrix

$=$ Buckingham $-\pi$-based moments

$=$ propeller force coefficient

$=$ propeller moment coefficient

$=$ Mach number

$=$ Buckingham- $\pi$-based moments

$=$ mass

$=$ Reynolds number

$=$ position of $A$ with respect to $B$

$=$ reference wing surface

$=N \times N$ symmetric positive definite matrices set

$=$ thrust force

$=$ left thrust force

$=$ right thrust force

$=$ linear velocity

$=$ freestream velocity

$=$ angle of attack

$=$ sideslip angle

= left elevon deflection

$=$ right elevon deflection

$=\phi$-theory elevon effectiveness

$=$ aerodynamic twist

$=$ freestream air density

$=\quad i$ th maximum singular value

$\tau \in \mathbb{R}^{6}$
$\Phi \in \mathbb{R}^{6 \times 6}$
$\Phi^{(f v)} \in \mathbb{R}^{3 \times 3}$
$\Phi^{(f \omega)} \in \mathbb{R}^{3 \times 3}$
$\Phi^{(m v)} \in \mathbb{R}^{3 \times 3}$
$\Phi^{(m \omega)} \in \mathbb{R}^{3 \times 3}$
$\phi \in \mathbb{R}_{+}$
$\omega \in \mathbb{R}^{3}$
$\omega_{1} \in \mathbb{R}$
$\omega_{2} \in \mathbb{R}^{\omega_{\infty} \in \mathbb{R}^{3}}$
$\omega^{a} \boldsymbol{\omega}^{b} \in \mathbb{R}^{3}$

$=$ aerodynamic wrench

$=\phi$-theory aerodynamic coefficient

$=\phi$-theory velocity-to-force coefficient

$=\phi$-theory angular-velocity-to-force coefficient

$=\phi$-theory velocity-to-moment coefficient

$=\phi$-theory angular-velocity-to-moment coefficient

$=\phi$-theory twist chord

$=$ inertial angular velocity

$=$ left propeller angular velocity

$=$ right propeller angular velocity

$=$ freestream angular velocity

$=$ angular velocity of $\mathcal{B}$ with respect to $\mathcal{A}$

\section{Introduction}

$\mathbf{T}$ HE past decade has seen rapid development of unmanned aerial vehicles (UAVs). Numerous different architectures are available for fulfilling a myriad of distinct, and often conflicting, purposes. Until very recently, most commercial UAVs were categorized as either fixed wing or rotary wing (multicopters). Although fixed-wing designs are known for their substantial endurance, rotary wings are notable for their hovering capabilities. A third category aiming at bridging those incompatible features has recently caught the attention of scientists and engineers, namely hybrid aerial vehicles (HAVs). Even though HAVs are long established (e.g., the 1963 early works regarding tilt-wing vehicles [1]), piloting early designs was demanding [2] and unsafe [3], thus precluding wide commercial viability. Recent advances in low-cost inertial sensors and embedded computing boosted the development of stabilizing control systems that allowed for stable and safe HAV piloting. Indeed, micro-HAVs are starting to find their way into consumer and business markets, e.g., the off-the-shelf Parrot Swing and Wingtra's WingtraOne, respectively.

From the point of view of HAV modeling for control design in Refs. [4,5], for instance, lookup tables were employed, which were obtained by means of typically lengthy static wind-tunnel campaigns, to design gain-scheduled controllers without resorting to a global mathematical model. On the other hand, in Ref. [6], wind-tunnel data were fitted to a given aerodynamic coefficient formula as a function of angle of attack $\alpha$ to obtain analytical models. Additionally, dynamic coefficients called for even more complex wind-tunnel campaigns or complex flight instrumentation [7]. If low-cost instrumentation was applied and data synchronization was prohibitively expensive, recent work [8] proposed a method for dynamic coefficient identification from flight data with unknown clock (timing) skews. Furthermore, not only parametric identification took place in modeling. For instance, Ref. [9] discussed methods for

\footnotetext{
*Ph.D. Candidate, Department of Aerospace Vehicles Design and Control. ${ }^{\dagger}$ Researcher, Department of Aerospace Vehicles Design and Control.

${ }_{\ddagger}$ Professor, Department of Aerodynamics, Energetics and Propulsion.
} 
measuring uncertainties due to the assumed aerodynamics coefficients algebraic model form.

This paper employs a steady-state approach to modeling aerodynamic coefficients. Dynamic vortex-shedding processes [10] are neglected, in which a rapid increase in the angle of attack causes the formation of a leading-edge vortex that produces an unsteady increase in lift. In this scenario, aerodynamic coefficients are dependent on the past history of angles of attack and sideslip.

Most fixed-wing controllers found in the literature decouple lateral and longitudinal dynamics so that separate modeling and control design take place independently. However, Ref. [11] analyzed the coupling effects between the lateral and longitudinal dynamics of a tilt-body vehicle (TBV) and concluded that unstable spiral dynamics were incorrectly rendered stable by decoupling assumptions. This suggested that coupled dynamics were fundamental for HAVs, and multiple-input multiple-output control design and modeling should be favored.

With the advent of fast and reliable embedded computing systems, algorithmic control theory [12] has been in the spotlight in recent years. This theory departs from the usual analytic-structure controller to a state-error-to-actuator mapping implicitly computed by an algorithm (often based on convex optimization). Recent examples related to HAVs include Refs. [13-15]. Reference [15], for instance, employed a multipurpose optimization tool to search for feasible tiltbody cruise-to-hover trajectories while optimizing a certain cost function. The present paper promotes this line of thought by providing, in addition to other features, an optimization-friendly dynamic model of HAVs. Efficient optimization solvers demand some sort of structure in the vehicle mathematical model, and HAV models are too general to fit solvers in an efficient (and thus real-time) fashion.

In addition to control design, optimization techniques allow for efficient aerodynamics model identification, such as in Ref. [16], in which a technique was proposed for postflight wind estimation in the absence of air-data sensors. Once the wind speed was estimated, flight data were corrected and used to perform aerodynamic coefficient estimation. Notice that, if efficient optimization routines were available, this same technique could be applied in real time for in-flight wind perturbation compensation.

From the propulsion modeling point of view, although obliqueflow aspects are crucial for flight performance analysis, their relevance to feedback control design is disputable. Furthermore, incidence propeller modeling is intricate and often requires windtunnel campaigns. For instance, Ref. [17] showed that, although the traditional combination of the blade element momentum theory, the vortex lattice method, and the momentum theory for oblique flow was a commonplace tool in helicopter design, it failed to properly reflect wind-tunnel data in high angles of incidence. The present paper further explores this issue in view of feedforward and feedback control design by means of a wind-tunnel campaign.

Finally, in this paper, flexible structural dynamic modes are not considered, and the reader is referred to Ref. [18] for a recent flexible flying-wing case study. In the present work, flexibility effects have not been observed during wind-tunnel testing in nominal HAV flight. Notice, however, that these effects might define the flight envelope itself. For example, Refs. $[19,20]$ acknowledged that cruise speed and range were limited directly or indirectly by proprotor/pylon/wing structural stability, known as whirl flutter, which is an important design consideration for tiltrotor aircraft.

The main contribution of this paper is a polynomial-like model structure that is fit for online optimization purposes yet complex enough to portray all intricacies of HAV flight. The authors call $\phi$ theory the ensemble of model equations and properties. In $\phi$ theory, modeling accuracy and simplicity are balanced. This provides an adequate description for global algorithmic guidance and control strategies. The remainder of the paper is organized as follows: Sec. II sets up notation and Sec. III explains the foundations of aerodynamic forces and moments modeling in light of the proposed $\phi$ theory, whereas Secs. IV, V, and VI show how to apply the aforementioned theory to model airfoils, wing-propeller interactions, and tail sitters, respectively. Section VII employs the model to 1) predict the theoretical existence and number of equilibrium longitudinal flight configurations, 2) compute trim points by means of a powerful algorithm developed herein, and 3) compare them to wind-tunnel data. Finally, Sec. VIII concludes the paper and highlights interesting opportunities arising out of this study.

\section{Notation}

In this paper, kinematic quantities of interest in multiple moving reference frames are rigorously studied; consequently, a consistent and precise notation is called for. Accordingly, this section outlines notation conventions including the definition of relevant reference frames and related variables of interest.

The notation ${ }^{a} \boldsymbol{x}^{c}$ is employed, where the symbol $x$ denotes the desired vector quantity (e.g., $p$ for position, $v$ for velocity, $a$ for acceleration, and $\omega$ for angular velocity) of point/frame (depending on the context) $\mathcal{C}$ with respect to frame $\mathcal{A}$. For instance, ${ }^{i} \boldsymbol{\omega}^{b}$ denotes the angular velocity of frame $\mathcal{B}$ with respect to frame $\mathcal{I}$. Additionally, the decomposition of a vector $x \in \mathbb{R}^{n}$ into its components in frame $\mathcal{R}$ is denoted by means of the right subscript position, e.g.,

$$
\boldsymbol{x}_{r}=\left(\begin{array}{llll}
x_{r 1} & x_{r 2} & \cdots & x_{r n}
\end{array}\right)^{T}
$$

All reference frames $\mathcal{F}$ of interest in this paper, unless otherwise stated, are right-handed and orthonormal, and hence isomorphic to the well-known $\mathrm{SO}(3)$ group, where

$$
\operatorname{SO}(3)=\left\{D \in \mathbb{R}^{3 \times 3}: D D^{T}=I, \operatorname{det} D=+1\right\}
$$

The vector product operation matrix representation $\left[\boldsymbol{v}_{b} \times\right] \in \mathfrak{s} \mathfrak{D}(3)$ (in some basis $\mathcal{B}$ ) is denoted by

$$
\boldsymbol{v}_{b} \times=\left[\boldsymbol{v}_{b} \times\right]=\left[\begin{array}{ccc}
0 & -v_{b 3} & v_{b 2} \\
v_{b 3} & 0 & -v_{b 1} \\
-v_{b 2} & v_{b 1} & 0
\end{array}\right]
$$

The quaternion algebra structure definition varies to a small extent in the literature. Herein, the adopted quaternion algebra $(\mathbb{Q}, \times)$ is defined as [21]

$$
\mathbb{Q}=\left\{q \in \mathbb{R}^{4}:\langle q, q\rangle=1\right\}
$$

and it is equipped with the quaternion product operation $\times: \mathbb{Q} \times \mathbb{Q} \rightarrow$ $\mathbb{Q}$ defined as

$$
p \times q=\left(\begin{array}{c}
p_{0} q_{0}-\boldsymbol{p}_{1} \cdot \boldsymbol{q}_{1} \\
p_{0} \boldsymbol{q}_{1}+q_{0} \boldsymbol{p}_{1}+\boldsymbol{p}_{1} \times \boldsymbol{q}_{1}
\end{array}\right)
$$

where

$$
p=\left(\begin{array}{c}
p_{0} \\
\boldsymbol{p}_{1}
\end{array}\right) \text { and } q=\left(\begin{array}{c}
q_{0} \\
\boldsymbol{q}_{1}
\end{array}\right) \text { with } p_{0}, q_{0} \in \mathbb{R}, \quad \boldsymbol{p}_{1}, \boldsymbol{q}_{1} \in \mathbb{R}^{3}
$$

The quaternion and direction cosine matrix from frames $\mathcal{A}$ to $\mathcal{B}$, respectively $\left(q_{b}^{a}\right.$ and $\left.D_{b}^{a}\right)$ are denoted such that their respective rotation formulas [21] are written as

$$
\left(\begin{array}{c}
0 \\
\boldsymbol{x}_{b}
\end{array}\right)=\left(q_{b}^{a}\right)^{\prime} \times\left(\begin{array}{c}
0 \\
\boldsymbol{x}_{a}
\end{array}\right) \times q_{b}^{a}
$$

and

$$
\boldsymbol{x}_{b}=D_{b}^{a} \boldsymbol{x}_{a}
$$

where $\left(q_{b}^{a}\right)^{\prime}$ denotes the quaternion conjugate of $q_{b}^{a}$. Finally, $\mathcal{L}$ and $\mathcal{B}$ denote, respectively, the local (presumed as also inertial) and body frames. 


\section{A. The Classic Approach}

The ubiquitous Buckingham- $\pi$-theorem-based approach [22] to modeling steady aerodynamic forces $\boldsymbol{F}_{b}^{\prime} \in \mathbb{R}^{3}$ and moments $\boldsymbol{M}_{b}^{\prime} \in \mathbb{R}^{3}$ on a finite wing $\mathcal{B}$ immersed in a uniform airflow with freestream velocity $\boldsymbol{v}_{\infty} \in \mathbb{R}^{3}$ is traditionally given by

$$
\boldsymbol{F}_{b}^{\prime}=\frac{1}{2} \rho S v_{\infty}^{2} \boldsymbol{C}_{b}(\alpha, \beta, M, R e)
$$

and

$$
\boldsymbol{M}_{b}^{\prime}=\frac{1}{2} \rho S v_{\infty}^{2} B \boldsymbol{K}_{b}(\alpha, \beta, M, R e)
$$

where

$$
B=\left[\begin{array}{lll}
b & 0 & 0 \\
0 & c & 0 \\
0 & 0 & b
\end{array}\right]
$$

and $b, c, \rho$, and $S \in \mathbb{R}_{+}^{*}$ denote, respectively, the reference wingspan, reference chord, freestream air density, and reference finite wing surface; and $\boldsymbol{C}_{b}$ and $\boldsymbol{K}_{b} \in \mathbb{R}^{3}$ denote the aerodynamic force and moment three-dimensional coefficients described in body frame $\mathcal{B}$. The latter are functions of the dimensionless quantities $\alpha$, $\beta, M$, and $R e$ : namely, the angle of attack, sideslip angle, and Mach and Reynolds numbers, respectively. Hereafter, airflow is assumed to be incompressible and inviscid; therefore, $\boldsymbol{C}_{b}$ and $\boldsymbol{K}_{b}$ are solely a function of $\alpha$ and $\beta$ during all flight conditions of interest. $\boldsymbol{C}_{b}(\alpha, \beta)$ and $\boldsymbol{K}_{b}(\alpha, \beta)$ are often (e.g., Refs. [23-25]) modeled as finite truncated sums of Fourier or Maclaurin series to fit wind-tunnel and flight data.

By contrast, $\phi$ theory proposes a novel $\{\alpha, \beta\}$-free parametrization of aerodynamic forces and moments. To understand the motivation behind this approach, recall that (see Fig. $\underline{1}$ for body $\mathcal{B}$ axes definition)

$$
\alpha=\tan ^{-1}\left(\frac{v_{\infty, b 3}}{v_{\infty, b 1}}\right)
$$

and

$$
\beta=\sin ^{-1}\left(\frac{v_{\infty, b 2}}{v_{\infty}}\right)
$$

Tail sitting and highly maneuverable flight, however, encounter near-zero freestream velocities (predominantly in wing sections not covered by propeller slipstream) that call for near-zero algebraic divisions in Eq. (12) and preclude the numerical stability of digital simulations.

Additionally, the present paper attempts to fit local experimental data while simultaneously striving for satisfactory qualitative global behavior. For instance, notice that a tail sitter in hover descent encounters an extrinsic $\alpha=\pi$ in view of traditional airfoil aerodynamics and calls for coherent aerodynamic coefficient extrapolation capabilities.

Finally, recent efforts [26] in semidefinite programming (SDP) and sums-of-squares (SOS) optimization allowed for efficient trajectory optimization and control in dynamic models governed by polynomial differential equations. The traditional aerodynamic coefficients formulation carries trigonometric functions that preclude SDP

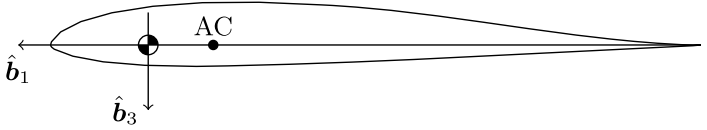

Fig. 1 MH45 airfoil with associated thin airfoil approximated aerodynamic center (AC). employment and call for computationally more expensive pathplanning routines. Alternatively, $\phi$ theory proposes an SDP-friendly formulation.

\section{B. $\phi$-Theory Parametrization}

This paper proposes a novel formulation, called $\phi$ theory, which is built upon the following $\{\alpha, \beta\}$-free parametrization of aerodynamic forces and moments:

$$
\boldsymbol{\tau}_{b}=-\frac{1}{2} \rho \operatorname{S\eta } C \Phi\left(\boldsymbol{\eta}_{b}\right) C \boldsymbol{\eta}_{b}
$$

where $\boldsymbol{\tau}_{b}, \boldsymbol{\eta}_{b} \in \mathbb{R}^{6}$, and $\eta \in \mathbb{R}_{+}$are, respectively, the aerodynamic wrench (with respect to the center of mass), wing twist, and aerodynamic $\phi$ norm given by

$$
\begin{gathered}
\boldsymbol{\tau}_{b}=\left(\begin{array}{ll}
\boldsymbol{F}_{b}^{T} & \boldsymbol{M}_{b}^{T}
\end{array}\right)^{T} \\
\boldsymbol{\eta}_{b}=\left(\begin{array}{ll}
\boldsymbol{v}_{\infty, b}^{T} & \boldsymbol{\omega}_{\infty, b}^{T}
\end{array}\right)^{T}
\end{gathered}
$$

and

$$
\eta=\sqrt{v_{\infty}^{2}+\phi \omega_{\infty}^{2}}, \quad \phi>0
$$

where $\phi \in \mathbb{R}_{+}^{*}$ is a dimensionless tunable parameter. The motivation for this formulation will become clear as its properties are studied in the following. Furthermore, $\omega_{\infty, b} \in \mathbb{R}^{3}$ denotes half the freestream vorticity $\xi_{\infty}$ such that

$$
\omega_{\infty}=\frac{1}{2} \xi_{\infty}=\frac{1}{2} \times v_{\infty}
$$

and $\phi \in \mathbb{R}_{+}^{*}$ is a tunable parameter. Note that $\omega_{\infty}$ is defined by means of vorticity for the sake of clarity. Nevertheless, although fluid, freestream flow constitutes a rigid motion and one can think of $\omega_{\infty}$ as the freestream angular velocity. In the absence of wind, one can easily prove that $\omega_{\infty}$ is equal to the vehicle angular velocity. Additionally, the wing screw reference matrix $C \in \mathbb{R}^{6 \times 6}$ is an extension of the wing reference matrix concept and is defined as

$$
C=\left[\begin{array}{cc}
I_{3 \times 3} & 0_{3 \times 3} \\
0_{3 \times 3} & B
\end{array}\right]
$$

Finally, $\Phi: \mathbb{R}^{6} \rightarrow \mathbb{R}^{6 \times 6}$ is the aerodynamic $\phi$ coefficient. The symbol $\phi$ is introduced in the nomenclature to facilitate parallels between the novel parametrization and the traditional Buckingham$\pi$-based coefficients/derivatives. Additionally, for the sake of brevity, Buckingham- $\pi$-based formulas and coefficients (e.g., $C_{L}$ and $C_{D}$ ) are referenced as $\pi$ theory and $\pi$ coefficients in the following, whereas the present model is referenced as $\phi$ theory and $\phi$ coefficients.

Unless otherwise stated, $\Phi(\cdot)$ is hereafter considered a constant function written as

$$
\Phi=\left[\begin{array}{ll}
\Phi^{(f v)} & \Phi^{(f \omega)} \\
\Phi^{(m v)} & \Phi^{(m \omega)}
\end{array}\right]
$$

where $\Phi^{(f v)}, \Phi^{(f \omega)}, \Phi^{(m v)}$, and $\Phi^{(m \omega)} \in \mathbb{R}^{3 \times 3}$. It will be presently shown that such an assumption greatly simplifies the model and yet still captures dominant features (e.g., poststall effects, aerodynamic derivatives, and global dissipation of energy) over the entire flight envelope (i.e., hover, cruise, and transition flight modes).

To avoid parameter clutter, $(1 / 2) \rho S C \Phi C$ will occasionally be simply denoted as $\Phi$ by abuse of notation. The meaning of $\Phi$ in any following occurrence should be clear from the context (especially by unit inspection). This consideration simplifies Eq. (14) to read

$$
\tau=-\eta \Phi \eta
$$


Some theoretical results follow to support $\phi$-theory employment in wide envelope applications. Next, this paper's main motivation (namely, the tilt-body vehicle problem) is addressed.

Definition III.1 (weightless body system): Let $\mathcal{B}$ be a rigid-body with inertia $(m, J)$ under the influence of aerodynamic forces and moments in a negligible gravity field with no wind. The weightless body dynamic system (WBS) is defined as the associated dynamical system given by

$$
\left\{\begin{array}{l}
\dot{\boldsymbol{v}}_{b}=\frac{1}{m} \boldsymbol{F}_{b}-\left[\boldsymbol{\omega}_{b} \times\right] \boldsymbol{v}_{b} \\
\dot{\boldsymbol{\omega}}_{b}=J^{-1} \boldsymbol{M}_{b}-J^{-1}\left[\boldsymbol{\omega}_{b} \times\right] J \boldsymbol{\omega}_{b} \\
\boldsymbol{\tau}_{b}=-\eta \Phi \boldsymbol{\eta}_{b}
\end{array}\right.
$$

Definition III.2 (freefall system): Let $\mathcal{B}$ be a rigid body with inertia $(m, J)$ by influence of aerodynamic forces and moments in a constant (with regard to the inertial frame $\mathcal{L}$ ) gravity field $\boldsymbol{g}_{l} \neq \mathbf{0}$ with no wind. The freefall dynamic system (FFS) is defined as the associated dynamical system given by

$$
\left\{\begin{array}{l}
\dot{\boldsymbol{v}}_{b}=\frac{1}{m} \boldsymbol{F}_{b}+D_{b}^{l} \boldsymbol{g}_{l}-\left[\boldsymbol{\omega}_{b} \times\right] \boldsymbol{v}_{b} \\
\dot{\boldsymbol{\omega}}_{b}=J^{-1} \boldsymbol{M}_{b}-J^{-1}\left[\boldsymbol{\omega}_{b} \times\right] J \boldsymbol{\omega}_{b} \\
\dot{D}_{b}^{l}=-\left[\boldsymbol{\omega}_{b} \times\right] D_{b}^{l} \\
\boldsymbol{\tau}_{b}=-\eta \Phi \boldsymbol{\eta}_{b}
\end{array}\right.
$$

Definition III.3 (terminal states and terminal velocities): The set of terminal states $\mathbb{T} \subset \mathbb{R}^{3} \times \mathbb{R}^{3} \times \mathrm{SO}(3)$ of a freefall dynamic system is defined as

$$
\mathbb{T}=\left\{\left(\boldsymbol{v}_{b}, \boldsymbol{\omega}_{b}, D_{b}^{l}\right) \in \mathbb{R}^{3} \times \mathbb{R}^{3} \times \operatorname{SO}(3): \dot{\boldsymbol{v}}_{b}=\mathbf{0}, \dot{\boldsymbol{\omega}}_{b}=\mathbf{0}, \dot{D}_{b}^{l}=0_{3 \times 3}\right\}
$$

Correspondingly, the set of terminal velocities $\mathbb{T}_{v} \subset \mathbb{R}^{3}$ is defined as

$$
\mathbb{T}_{v}=\left\{\boldsymbol{v}_{b} \in \mathbb{R}^{3}:\left(\boldsymbol{v}_{b}, \boldsymbol{\omega}_{b}, D_{b}^{l}\right) \in \mathbb{T} \text { for some } \boldsymbol{\omega}_{b}, D_{b}^{l}\right\}
$$

Theorem III.1 (dissipative aerodynamics): Assume a WBS with arbitrary initial conditions $\boldsymbol{v}_{b}(0), \omega_{b}(0) \in \mathbb{R}^{3}$. If $\Phi>0$, then $\boldsymbol{v}_{b}(t) \rightarrow \mathbf{0}$ and $\boldsymbol{\omega}_{b}(t) \rightarrow \mathbf{0}$ uniformly as $t \rightarrow \infty$.

Proof: Consider the Lyapunov candidate function $V(\boldsymbol{\eta}): \mathbb{R}^{6} \rightarrow \mathbb{R}_{+}$ given by

$$
V\left(\boldsymbol{v}_{b}, \boldsymbol{\omega}_{b}\right)=\frac{1}{2} m \boldsymbol{v}_{b}^{T} \boldsymbol{v}_{b}+\frac{1}{2} \boldsymbol{\omega}_{b}^{T} J \boldsymbol{\omega}_{b}
$$

Notice that

$$
V(\boldsymbol{\eta}) \leq \frac{m}{2} v_{b}^{2}+\frac{\sigma_{1}(J)}{2} \omega_{b}^{2} \leq \frac{1}{2} \max \left\{m, \sigma_{1}(J)\right\} \eta^{2}
$$

and therefore $V(\boldsymbol{\eta})$ is decrescent $\left[\sigma_{1}(\cdot)\right.$ denotes the maximum singular value]. A similar development using the minimum singular value allows one to prove that $V(\boldsymbol{\eta})$ is positive definite. Differentiation with respect to time yields

$$
\dot{V}(\boldsymbol{\eta})=m \boldsymbol{v}_{b}^{T} \frac{\mathrm{d}}{\mathrm{d} t} \boldsymbol{v}_{b}+\boldsymbol{\omega}_{b}^{T} J \frac{\mathrm{d}}{\mathrm{d} t} \boldsymbol{\omega}_{b}
$$

because $J=J^{T}$ for every inertia matrix. Substitution of Eqs. (14) and (22) into Eq. (28) yields

$$
\begin{aligned}
& \dot{V}(\boldsymbol{\eta})=-\boldsymbol{v}_{b}^{T} \frac{1}{2} \rho S \eta\left[\begin{array}{ll}
\Phi^{(f v)} & \Phi^{(f \omega)}
\end{array}\right]\left(\begin{array}{c}
\boldsymbol{v}_{b} \\
B \boldsymbol{\omega}_{b}
\end{array}\right) \\
& -\boldsymbol{\omega}_{b}^{T} \frac{1}{2} \rho S \eta B\left[\begin{array}{ll}
\Phi^{(m v)} & \Phi^{(m \omega)}
\end{array}\right]\left(\begin{array}{c}
\boldsymbol{v}_{b} \\
B \boldsymbol{\omega}_{b}
\end{array}\right)+ \\
& -\underbrace{m \boldsymbol{v}_{b}^{T}\left[\boldsymbol{\omega}_{b} \times\right] \boldsymbol{v}_{b}}_{\mathbf{0}}-\underbrace{\boldsymbol{\omega}_{b}^{T}\left[\boldsymbol{\omega}_{b} \times\right] J \boldsymbol{\omega}_{b}}_{\mathbf{0}}=-\frac{1}{2} \rho S \eta \boldsymbol{\eta}^{T} \Phi \boldsymbol{\eta}<0 \quad \forall \boldsymbol{\eta} \neq \mathbf{0}
\end{aligned}
$$

allowing one to conclude by the Lyapunov method that $\boldsymbol{v}_{b}(t) \rightarrow \mathbf{0}$ and $\boldsymbol{\omega}_{b}(t) \rightarrow \mathbf{0}$ uniformly as $t \rightarrow+\infty$.

Notice that Theorem III. 1 corroborates $\phi$-theory consistency in wide envelope applications if appropriate $\Phi \in S_{++}^{6}$ are chosen $\left(S_{++}^{n}\right.$ is the set of symmetric positive definite matrices in $\left.\mathbb{R}^{n \times n}\right)$. Aerodynamic wrenches are dissipative in reality, and Theorem III.1 provides a sufficient condition on $\Phi$ for achieving $\dot{V} \leq 0$ globally. Similarly, the following results explore additional $\phi$-theory properties.

Lemma III.1 (geometry of terminal states): Assume an FFS with $\Phi>0$. If $\Phi^{(m v)}$ is full rank, then $\mathbb{T}=\varnothing$. Otherwise, $\left(\boldsymbol{v}_{0}, \omega_{0}, D_{0}\right) \in \mathbb{T}$ if, and only if,

$$
\left\{\begin{array}{l}
\boldsymbol{\omega}_{0}=\mathbf{0}, \boldsymbol{v}_{0} \neq \mathbf{0} \\
\hat{\boldsymbol{v}}_{0} \in \operatorname{ker}\left(\Phi^{(m v)}\right) \\
v_{0}=\sqrt{\frac{2 m g}{\rho S\left\|\Phi^{(f v)} \hat{\boldsymbol{v}}_{0}\right\|}} \\
\frac{1}{\left\|\Phi^{(f v)} \hat{\boldsymbol{v}}_{0}\right\|} \Phi^{(f v)} \hat{\boldsymbol{v}}_{0}=-D_{0} \hat{\boldsymbol{g}}_{l}
\end{array}\right.
$$

Proof: By definition, the terminal states are the equilibrium points of Eq. (23), namely, the points $\left(\boldsymbol{v}_{b}, \boldsymbol{\omega}_{b}, D_{b}^{l}\right)$ such that

$$
\frac{1}{m} \boldsymbol{F}_{b}+D_{b}^{l} \boldsymbol{g}_{l}-\left[\boldsymbol{\omega}_{b} \times\right] \boldsymbol{v}_{b}=\mathbf{0}
$$

$$
\boldsymbol{M}_{b}-\left[\boldsymbol{\omega}_{b} \times\right] J \boldsymbol{\omega}_{b}=\mathbf{0}
$$

and

$$
-\left[\omega_{b} \times\right] D_{b}^{l}=0
$$

Because $D_{b}^{l}$ is nonsingular, Eq. (33) yields $\boldsymbol{\omega}_{b}=\mathbf{0}$ and simplifies Eqs. (31) and (32) to read

$$
\frac{1}{2 m} \rho S v_{b} \Phi^{(f v)} \boldsymbol{v}_{b}+D_{b}^{l} \boldsymbol{g}_{l}=\mathbf{0}
$$

and

$$
\frac{1}{2} \rho S B v_{b} \Phi^{(m v)} \boldsymbol{v}_{b}=\mathbf{0}
$$

Notice that $\boldsymbol{v}_{b}=\mathbf{0}$ does not compose a terminal state due to Eq. (34). Therefore, if $\operatorname{rank}\left(\Phi^{(m v)}\right)=3$, then $\Phi^{(m v)}$ is full rank and Eq. ( $(\overline{35})$ allows only for $\boldsymbol{v}_{b}=\mathbf{0}$, and thus $\mathbb{T}=\varnothing$.

On the other hand, if $\operatorname{rank}\left(\Phi^{(m v)}\right) \leq 2$, the solution of Eq. (35) is the linear subspace $\operatorname{ker}\left(\Phi^{(m v)}\right) \subset \mathbb{R}^{3}$. Let $\hat{\boldsymbol{w}} \in \operatorname{ker}\left(\Phi^{(m v)}\right)$ be an arbitrary direction in $\operatorname{ker}\left(\Phi^{(m v)}\right)$. Notice that $w \hat{\boldsymbol{w}}$ can always be made a solution of Eq. (34) by choosing

$$
w=\sqrt{\frac{2 m g}{\rho S\left\|\Phi^{(f v)} \hat{\boldsymbol{w}}\right\|}}
$$


and an appropriate rotation $D_{0}$ (notice that it always exists, and there are uncountably infinite choices) that rotates $\hat{\boldsymbol{g}}_{l}$ in the direction of $\Phi^{(f v)} \hat{\boldsymbol{w}}$; that is,

$$
\frac{1}{\left\|\Phi^{(f v)} \hat{\boldsymbol{w}}\right\|} \Phi^{(f v)} \hat{\boldsymbol{w}}=-D_{0} \hat{\boldsymbol{g}}_{l}
$$

Corollary III.1: Assume an FFS with $\Phi>0 . \mathbb{T}_{v}$ is either 1) empty, if $\operatorname{rank}\left(\Phi^{(m v)}\right)=3 ; 2$ ) a couple of antipodal points, if $\operatorname{rank}\left(\Phi^{(m v)}\right)=2$; 3 ) a closed planar curve in $\mathbb{R}^{3}$, if $\operatorname{rank}\left(\Phi^{(m v)}\right)=1$; or 4 ) a closed surface in $\mathbb{R}^{3}$, if $\operatorname{rank}\left(\Phi^{(m v)}\right)=0$.

Figure 2 illustrates Corollary III.1. Notice how geometrical symmetries influence $\operatorname{rank}\left(\Phi^{(m v)}\right)$. For instance, symmetry around a point (i.e., a ball) suggests $\operatorname{rank}\left(\Phi^{(m v)}\right)=0$, whereas $\operatorname{rank}\left(\Phi^{(m v)}\right)=$ 1 evokes symmetry around an axis (e.g., an ellipsoid). Additionally, the authors suggest $\operatorname{rank}\left(\Phi^{(m v)}\right)=2$ for modeling airfoils due to isolated equilibrium points. These remarks are paramount to ensuring adequate $\phi$ coefficients for a given application. Finally, $\operatorname{rank}\left(\Phi^{(m v)}\right)=3$ prohibits terminal states but carries interesting properties that are studied in the following.

Lemma III.2 (Uniform ultimate boundedness): Assume an FFS with an arbitrary initial condition $\boldsymbol{\eta}(0)=\boldsymbol{\eta}_{0} \in \mathbb{R}^{6}$. If $\Phi>0$, then there exists $t_{*} \in \mathbb{R}_{+}$such that

$$
|\boldsymbol{\eta}(t)| \leq \sqrt{\frac{2 m g}{\rho S \sigma_{6}(\Phi)} \frac{\max \left(m, \sigma_{1}(J)\right)}{\min \left(m, \sigma_{3}(J)\right)} \sqrt{\frac{\max (1, \phi)}{\min (1, \phi)}}}
$$

for all $t \geq t_{*}$.

Proof: Consider once more the Lyapunov candidate function given by Eq. (26). Similarly to Eq. (29), differentiation with respect to time yields

$$
\dot{V}(\boldsymbol{\eta})=-\frac{1}{2} \rho S \eta \boldsymbol{\eta}^{T} \Phi \boldsymbol{\eta}+m \boldsymbol{v}_{b}^{T} \boldsymbol{g}_{b}
$$

Additionally, notice that

$$
\sqrt{\min (1, \phi)} \sqrt{v_{b}^{2}+w_{b}^{2}} \leq \eta \leq \sqrt{\max (1, \phi)} \sqrt{v_{b}^{2}+w_{b}^{2}}
$$

and

$$
\alpha_{1}(|\boldsymbol{\eta}|) \leq V(\boldsymbol{\eta}) \leq \alpha_{2}(|\boldsymbol{\eta}|)
$$

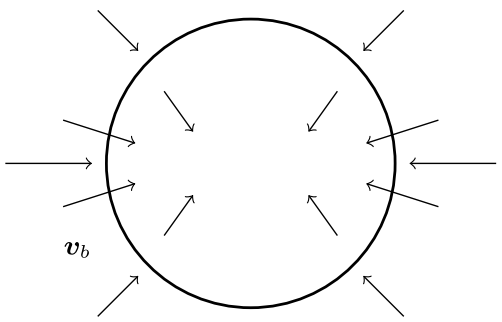

a) $\operatorname{ker}\left(\Phi^{(m v)}\right)=\mathbb{R}^{3}$

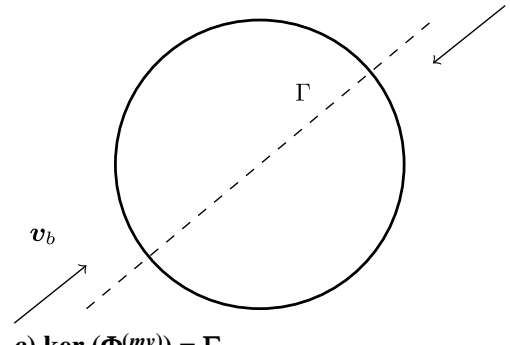

c) $\operatorname{ker}\left(\Phi^{(m v)}\right)=\Gamma$

Fig. 2 Admissible terminal $\mathbb{T}_{v}$ geometries in view of different $\Phi>0$. where $\alpha_{1}(\cdot)$ and $\alpha_{2}(\cdot) \in K_{\infty}$ are given according to Eq. (27). Observe that $\dot{V}(\boldsymbol{\eta})$ is not negative definitive in the presence of gravity. However, if

$$
|\boldsymbol{\eta}| \geq \underbrace{\sqrt{\frac{2 m g}{\rho S \sigma_{6}(\Phi)}}}_{\mu} \text { then } m g \leq \frac{|\boldsymbol{\eta}|^{2} \rho S \sigma_{6}(\Phi)}{2}
$$

where $\sigma_{6}(\Phi)$ is the smallest singular value of $\Phi$, and thus

$$
\begin{aligned}
\dot{V}(\boldsymbol{\eta}) & =-\frac{1}{2} \rho S \eta \boldsymbol{\eta}^{T} \Phi \boldsymbol{\eta}+m \boldsymbol{v}_{b}^{T} \boldsymbol{g}_{b} \\
& \leq-\frac{1}{2} \rho S|\boldsymbol{\eta}|^{3} \sigma_{6}(\Phi) \sqrt{\min (1, \phi)}+m|\boldsymbol{\eta}| g \sqrt{\max (1, \phi)}<0
\end{aligned}
$$

Therefore,

$$
S=\left\{\boldsymbol{\eta} \in \mathbb{R}^{6}:|\boldsymbol{\eta}| \leq \alpha_{1}^{-1}\left(\alpha_{2}(\mu)\right)\right\}
$$

is an invariant set and, by ultimate boundedness arguments, the lemma is proved because

$$
\alpha_{1}^{-1}\left(\alpha_{2}(\mu)\right)=\sqrt{\frac{2 m g}{\rho S \sigma_{6}(\Phi)} \frac{\max \left(m, \sigma_{1}(J)\right)}{\min \left(m, \sigma_{3}(J)\right)} \sqrt{\frac{\max (1, \phi)}{\min (1, \phi)}}}
$$

Lemma III. 2 bounds the velocity of falling objects and sheds light, for instance, on 1) the periodic exchange of linear kinetic and angular energies in falling leaves, and 2) the stability (in the sense of Lyapunov) of falling objects.

Notice that body aerodynamic coefficients are fully determined by one object, namely, $(\phi, \Phi) \in \mathbb{R}_{+}^{*} \times S_{++}^{6}$. For instance, $\Phi$ encapsulates information about airfoil coefficients $C_{l}, C_{d}, C_{y}, C_{l}$, $C_{m}$, and $C_{n}$, as detailed in Secs. IV.A and IV.B. Additionally, the fact that all physically meaningful coefficients $\Phi$ lay in $S_{++}^{6}$ allows for efficient identification algorithm implementation by means of positive semidefinite programming [26] optimization techniques. Furthermore, rigid-body differential equations of motion are polynomial-like and invite SOS optimization to take place for trajectory planning and control.

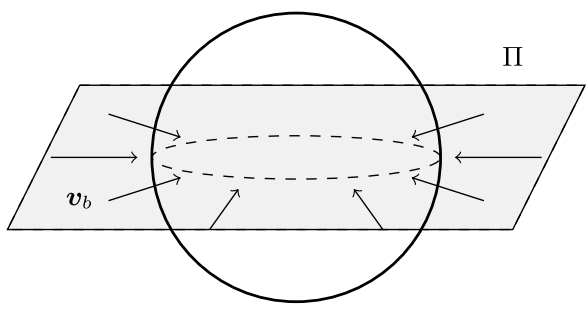

b) $\operatorname{ker}\left(\Phi^{(m v)}\right)=\Pi$

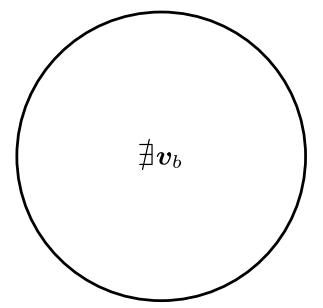

d) $\operatorname{ker}\left(\Phi^{(m v)}\right)=\{0\}$ 
Finally, notice that Eq. (14) does not cover all possibilities of $\pi$-theory formulation given by Eqs. (9) and (10). However, the following examples illustrate that numerous phenomena are qualitatively modeled by the $\phi$ approach, which ultimately allows for robust control design and numerical stable simulation of TBVs.

\section{Finite Wing Aerodynamic Coefficients}

\section{A. Airfoil Aerodynamic Static Coefficients}

To establish a parallel between $\phi$ and $\pi$ coefficients, consider an airfoil $\mathcal{B}$ immersed in an airflow of density $\rho$ with freestream velocity $\boldsymbol{v}_{\infty}$ at fixed angles of attack $\alpha$ and sideslip $\beta$ (thus, $\boldsymbol{\omega}_{\infty}=\mathbf{0}$ ). Application of the $\phi$ framework yields

$$
\boldsymbol{F}_{b}=-\frac{1}{2} \rho S v_{\infty} \Phi^{(f v)} \boldsymbol{v}_{\infty, b}
$$

where, for $x z$-symmetrical vehicles, $\Phi^{(f v)}$ is given by

$$
\Phi^{(f v)}=\left[\begin{array}{ccc}
\phi_{11}^{(f v)} & 0 & \phi_{13}^{(f v)} \\
0 & \phi_{22}^{(f v)} & 0 \\
\phi_{31}^{(f v)} & 0 & \phi_{33}^{(f v)}
\end{array}\right]
$$

Notice that $\phi_{21}^{(f v)}=\phi_{23}^{(f v)}=0$ because longitudinal flight should yield $F_{b 2}=0$ in view of vehicle $x z$ symmetry. Additionally, $\phi_{12}^{(f v)}=\phi_{21}^{(f v)}, \phi_{32}^{(f v)}=\phi_{23}^{(f v)}$, and $\phi_{31}^{(f v)}=\phi_{13}^{(f v)}$ due to matrix symmetry suggested by Theorem III.1. Manipulation of Eq. (45) yields

$$
\boldsymbol{F}_{b}=-\frac{1}{2} \rho S v_{\infty} \Phi^{(f v)} \boldsymbol{v}_{\infty, b}=-\frac{1}{2} \rho S v_{\infty}^{2} \Phi^{(f v)} \frac{\boldsymbol{v}_{\infty, b}}{v_{\infty}}
$$

and therefore

$$
\boldsymbol{C}_{b}(\alpha, \beta)=-\Phi^{(f v)} \frac{\boldsymbol{v}_{\infty, b}}{v_{\infty}}
$$

To render the traditional aerodynamic coefficients more explicit, Eq. (48) is rewritten as

$$
\left(\begin{array}{l}
C_{d}(\alpha, \beta) \\
C_{c}(\alpha, \beta) \\
C_{l}(\alpha, \beta)
\end{array}\right)=-C_{w}=D_{w}^{b} \Phi^{(f v)} \frac{\boldsymbol{v}_{\infty, b}}{v_{\infty}}
$$

where $\mathcal{W}$ denotes the wind reference frame. In longitudinal flight, Eq. (49) yields

$$
-C_{w}=\left[\begin{array}{ccc}
c \alpha & 0 & s \alpha \\
0 & 1 & 0 \\
-s \alpha & 0 & c \alpha
\end{array}\right]\left[\begin{array}{ccc}
\phi_{11}^{(f v)} & 0 & \phi_{13}^{(f v)} \\
0 & \phi_{22}^{(f v)} & 0 \\
\phi_{13}^{(f v)} & 0 & \phi_{33}^{(f v)}
\end{array}\right]\left(\begin{array}{c}
\cos \alpha \\
0 \\
\sin \alpha
\end{array}\right)
$$

thus

$$
-C_{w}=\left(\begin{array}{c}
\phi_{11}^{(f v)} c^{2} \alpha+2 \phi_{13}^{(f v)} s \alpha c \alpha+\phi_{33}^{(f v)} s^{2} \alpha \\
0 \\
\phi_{13}^{(f v)} c^{2} \alpha+\left(\phi_{33}^{(f v)}-\phi_{11}^{(f v)}\right) s \alpha c \alpha-\phi_{13}^{(f v)} s^{2} \alpha
\end{array}\right)
$$

Because $\sin ^{2} \alpha+\cos ^{2} \alpha=1$, Eq. ( $\underline{51}$ ) can be rewritten as

$$
\left(\begin{array}{c}
C_{d} \\
C_{l}
\end{array}\right)=\left[\begin{array}{cc}
\phi_{11}^{(f v)}-\phi_{33}^{(f v)} & 2 \phi_{13}^{(f v)} \\
2 \phi_{13}^{(f v)} & \phi_{33}^{(f v)}-\phi_{11}^{(f v)}
\end{array}\right]\left(\begin{array}{c}
c^{2} \alpha \\
s \alpha c \alpha
\end{array}\right)+\left(\begin{array}{c}
\phi_{33}^{(f v)} \\
-\phi_{13}^{(f v)}
\end{array}\right)
$$

which by means of the following double-arc formulas

$$
\left(\begin{array}{c}
\cos ^{2} \alpha \\
\sin \alpha \cos \alpha
\end{array}\right)=\frac{1}{2}\left(\begin{array}{c}
\cos 2 \alpha \\
\sin 2 \alpha
\end{array}\right)+\left(\begin{array}{c}
\frac{1}{2} \\
0
\end{array}\right)(53)
$$

one obtains

$$
\begin{aligned}
& \left(\begin{array}{c}
C_{d} \\
C_{l}
\end{array}\right)=\underbrace{\frac{1}{2}\left[\begin{array}{cc}
\phi_{11}^{(f v)}-\phi_{33}^{(f v)} & 2 \phi_{13}^{(f v)} \\
2 \phi_{13}^{(f v)} & \phi_{33}^{(f v)}-\phi_{11}^{(f v)}
\end{array}\right]}_{A_{\phi}=\left[\boldsymbol{a}_{\phi 1} \boldsymbol{a}_{\phi 2}\right]}\left(\begin{array}{c}
c 2 \alpha \\
s 2 \alpha
\end{array}\right) \\
& +\underbrace{\frac{1}{2}\left(\begin{array}{c}
\phi_{33}^{(f v)}+\phi_{11}^{(f v)} \\
0
\end{array}\right)}_{\boldsymbol{b}_{\phi}}
\end{aligned}
$$

Notice that Eq. (54) maps the unit circle to an ellipse $E_{\phi} \subset \mathbb{R}^{2}$ in the $\left(C_{d}, C_{l}\right)$ domain [double covered by $\left.\alpha \in[0,2 \pi)\right]$. Additionally, $A_{\phi}$ is orthogonal with column vectors denoted by $\boldsymbol{a}_{\phi 1}$ and $\boldsymbol{a}_{\phi 2}$ (i.e., $\left.A_{\phi}=\left[\begin{array}{ll}a_{\phi 1} & a_{\phi 2}\end{array}\right]\right)$. These properties allow for a direct drag polar sketch from inspection of $\Phi$, as Fig. 3 illustrates. For that, first identify $\boldsymbol{b}_{\phi}, \boldsymbol{a}_{\phi 1}$, and $\boldsymbol{a}_{\phi 2}$; and sketch the ellipse accordingly. Second, identify the force direction by drawing a $2 \alpha$ arc from $\boldsymbol{a}_{\phi 1}$ (counterclockwise). The aerodynamic force $\boldsymbol{F}_{w}$ is parallel to the line connecting the end of the arc to the origin. Conversely, wind-tunnel data can be used to identify $\phi$-model parameters by means of ellipse fitting.

Lemma IV.1: If $\Phi>0$ then $E_{\phi} \subset \mathbb{R}_{+}^{*} \times \mathbb{R} \times \mathbb{R}$.

Proof: Recall from Eq. (9) that

$$
\left(\begin{array}{l}
C_{d}(\alpha, \beta) \\
C_{c}(\alpha, \beta) \\
C_{l}(\alpha, \beta)
\end{array}\right)=D_{w}^{b} \Phi^{(f v)} \frac{v_{\infty, b}}{v_{\infty}}
$$

thus

$$
C_{d}(\alpha, \beta)=\left[\begin{array}{lll}
c \alpha c \beta & s \beta & s \alpha c \beta
\end{array}\right] \Phi^{(f v)}\left(\begin{array}{c}
c \alpha c \beta \\
s \beta \\
s \alpha c \beta
\end{array}\right)
$$

Because $\Phi>0$, then $\Phi^{(f v)}>0$; and by Eq. (56), one concludes that $C_{d}(\alpha, \beta)>0$ for all $\alpha, \beta \in \mathbb{R}$.
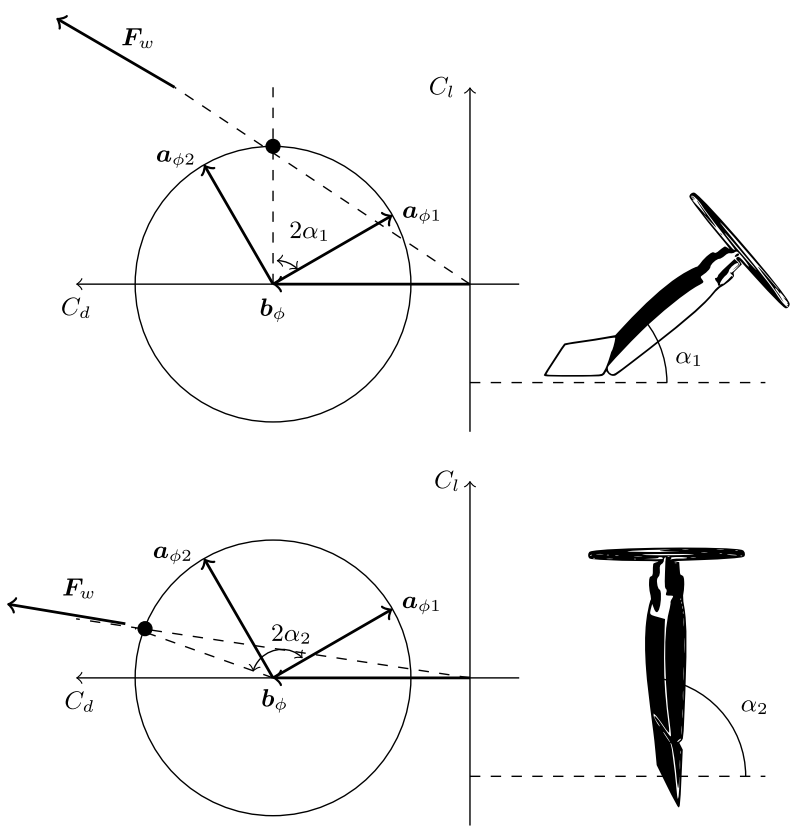

Fig. 3 Elliptical drag polar concept allowing for global qualitative visualization of aerodynamic forces for arbitrary angles of attack. Two examples are shown: transition and hover. 
In other words, Theorem III.1 and Lemma IV.1 state that physically meaningful drag polars modeled by $\phi$ theory must reside in the right-hand side of the $\left(C_{d}, C_{l}\right)$ domain.

Although a circular drag polar might appear overly restrictive, notice that an arbitrary drag polar can be bounded by two circles. Robustness studies might benefit from this simple and visual approach for addressing aerodynamic modeling uncertainties.

\section{B. Airfoil Aerodynamic Derivatives}

Although Sec. IV.A established a parallel between $\pi$-static coefficients (e.g., $\bar{C}_{l}$ and $C_{m}$ ) and $\Phi$, this section presents a connection between $\Phi$ and $\pi$ derivatives (e.g., $C_{l_{p}}, C_{m_{q}}$, and $C_{n_{r}}$ ). Accordingly, consider a finite wing $\mathcal{B}$ immersed in an airflow of density $\rho$ with freestream velocity $\boldsymbol{v}_{\infty}$ at time-varying angles of attack $\alpha(t)$ and sideslip $\beta(t)$. Application of $\phi$ theory yields

$$
\boldsymbol{M}_{b}=\frac{1}{2} \rho S B \sqrt{v_{\infty}^{2}+\phi \omega_{b}^{2}}\left(\Phi^{(m v)} \boldsymbol{v}_{b}+\Phi^{(m \omega)} B \boldsymbol{\omega}_{b}\right)
$$

Assuming longitudinal flight and $v_{b}^{2} \gg \phi \omega_{b}^{2}$ (a mild assumption for general aviation aircraft), Eq. (그) yields

$$
\boldsymbol{M}_{b} \approx \frac{1}{2} \rho S B v_{\infty}\left(\Phi^{(m v)} \boldsymbol{v}_{b}+\Phi^{(m \omega)} B \boldsymbol{\omega}_{b}\right)
$$

The first term is analogous to that in Sec. IV.A. The components of the second term (for instance, $M_{b 2}$ ) can be rewritten as

$$
M_{b 2}=\frac{1}{2} \rho S c v_{b}^{2}\left(\frac{c}{2 v_{b}}\right)(\underbrace{2 \phi_{21}^{(m \omega)}}_{C_{m_{p}}} P+\underbrace{2 \phi_{22}^{(m \omega)}}_{C_{m_{q}}} Q+\underbrace{2 \phi_{23}^{(m \omega)}}_{C_{m_{r}}} R)
$$

From inspection of Eq. (59), one concludes that $\phi$-theory and $\pi$-theory aerodynamic derivatives are related according to

$$
\Phi^{(m \omega)}=\frac{1}{2}\left[\begin{array}{lll}
C_{l_{p}} & C_{l_{q}} & C_{l_{r}} \\
C_{m_{p}} & C_{m_{q}} & C_{m_{r}} \\
C_{n_{p}} & C_{n_{q}} & C_{n_{r}}
\end{array}\right]
$$

Requiring $\Phi>0$ implies enforcing $\Phi^{(m \omega)}>0$, although substitution of $\pi$ coefficients in Eq. (60) might result in $\Phi^{(m \omega)} \notin S_{++}^{3}$. In such a case, the authors suggest exploiting the closed convex cone structure of $S_{++}^{3}$ to perform projections [27] in view of the usual inner product $\langle X, Y\rangle=\operatorname{tr}\left(X^{T} Y\right)$.

\section{Application: Thin Airfoils}

Classical thin symmetric airfoil theory [22] predicts $\partial C_{l} / \partial \alpha=2 \pi$ with an associated center of pressure located at a quarter-chord from the leading edge. Additionally, the pitching moment coefficient $C_{m}(\alpha)$ is proved to be identically zero at the center of pressure (and hence this point is the aerodynamic center). This section illustrates how $\phi$ theory can be applied to thin airfoil modeling by encoding the aforementioned properties into $\Phi$.

First, differentiation of Eq. (4ㅜ) with respect to $\alpha$ yields

$$
\left.\frac{\partial}{\partial \alpha}\left(\begin{array}{l}
C_{d} \\
C_{l}
\end{array}\right)\right|_{\alpha=0}=\left.2 A_{\phi}\left(\begin{array}{c}
-\sin 2 \alpha \\
\cos 2 \alpha
\end{array}\right)\right|_{\alpha=0}=\left(\begin{array}{c}
2 \phi_{13} \\
\phi_{33}-\phi_{11}
\end{array}\right)
$$

and thus the $2 \pi$ lift slope condition imposes

$$
\phi_{33}=2 \pi+\phi_{11}
$$

Furthermore, airfoil symmetry calls for $\partial C_{d} / \partial \alpha=0$ at $\alpha=0$. Therefore,

$$
\phi_{13}=0
$$

and

$$
\phi_{11}=C_{d 0}
$$

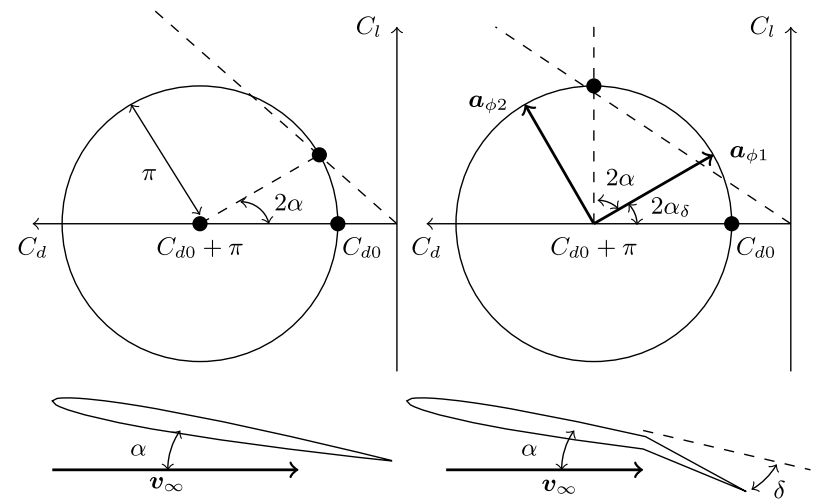

Fig. 4 Two thin symmetric airfoils: freestream velocity with angle of attack $\alpha$ applied (left), and aileron modeling achieved by means of a phase shift (right).

where $C_{d 0}$ denotes the minimum drag coefficient. In summary, for symmetrical thin airfoils,

$$
\Phi^{(f v)}=\left[\begin{array}{ccc}
C_{d 0} & 0 & 0 \\
0 & C_{y 0} & 0 \\
0 & 0 & 2 \pi+C_{d 0}
\end{array}\right]
$$

which is illustrated by the elliptical polar in Fig. 4. By inspection of Fig. 4, one concludes that stall occurs beyond $\alpha_{s}=45 \mathrm{deg}$ with an associated lift coefficient of $C_{l}\left(\alpha_{s}\right)=\pi$.

Cambered airfoil $\Phi^{(f v)}$ modeling is achieved by rotation of $\boldsymbol{a}_{\phi 1}$ and $\boldsymbol{a}_{\phi 2}$ by an angle $2 \alpha_{\delta}(\delta)$ (see Fig. 4 ). Because an increase in $\delta$ normally yields an increase in lift, $\overline{\alpha_{\delta}}(\cdot)$ is assumed continuous and monotonically increasing (for small $\delta$ ).

Second, $\Phi^{(m v)}$ is intimately connected to center-of-mass location with respect to the aerodynamic center. In Fig. $1, \Delta r<0$ and the center of mass is ahead of the aerodynamic center. The moment $\boldsymbol{M}_{b}$ with respect to the center of mass is therefore modeled by

$$
\boldsymbol{M}_{b}=\left[\boldsymbol{r}^{a c / c g} \times\right] \frac{1}{2} \rho S v_{\infty} \Phi^{(f v)} \boldsymbol{v}_{\infty, b}
$$

and thus

$$
\Phi^{(m v)}=B^{-1}\left[\boldsymbol{r}^{a c / c g} \times\right] \Phi^{(f v)}
$$

Often, $\boldsymbol{r}^{a c / c g}$ lies in the vehicle $\hat{\boldsymbol{x}}_{b}$ axis (i.e., $\boldsymbol{r}=\Delta \boldsymbol{r} \hat{\boldsymbol{b}}_{1}$ such that $\Delta r>0$ implies an aerodynamic center in front of the center of gravity), and therefore Eq. (67) simplifies to read

$$
\Phi^{(m v)}=\left[\begin{array}{ccc}
0 & 0 & 0 \\
0 & 0 & -c^{-1} \Delta r\left(2 \pi+C_{d 0}\right) \\
0 & b^{-1} \Delta r C_{y 0} & 0
\end{array}\right]
$$

Third, $\Phi>0$ implies $\phi_{i j}^{(f \omega)}=\phi_{j i}^{(m v)}$. Finally, $\Phi^{(m \omega)}$ is modeled as Eq. (60).

The striking correlation between $\Phi^{(m v)}$ and $\Phi^{(f \omega)}$ enforces conservation of energy. An accelerating pitching moment comes at the expense of a damping force, and vice versa. This reciprocity sheds light on how gravity forces sustain angular periodic motion back in a falling leaf, for example.

\section{Wing-Propeller Interaction}

A common approach to modeling wing-propeller interaction is by means of the propwash-induced velocity concept [28]. The fundamental idea is that the velocity field $v_{\infty}$ is disturbed by the propeller downstream such that its intensity $\left|\boldsymbol{v}_{\infty}\right|$ is increased, whereas $|\alpha|$ and $|\beta|$ are decreased. The ubiquitous approach is to resort to inviscid conservation of momentum arguments, which in its integral form [22] yields 


$$
\frac{\partial}{\partial t} \iiint_{V} \rho \boldsymbol{v} \mathrm{d} V+\oiint_{S(V)}(\rho \boldsymbol{v} \cdot \mathrm{d} \boldsymbol{S}) \boldsymbol{v}+\oiint_{S(V)} p \mathrm{~d} \boldsymbol{S}=\iiint_{V} \rho \boldsymbol{f} \mathrm{d} V
$$

Assuming incompressible and steady flow $v$, negligible body forces $f$, a propeller disk of area $S_{p}$, a control volume $V$ around the propeller disk, freestream velocity $\boldsymbol{v}_{\infty}$, and propwash vena contracta velocity $\boldsymbol{\psi}$, Eq. (69) yields

$$
\psi \boldsymbol{\psi}=v_{\infty} \boldsymbol{v}_{\infty}+\frac{1}{\rho S_{p}} \boldsymbol{T}
$$

For instance, in steady hover flight, Eq. (70) yields $\psi_{h}=\sqrt{T / \rho S_{p}}$. The square root operation in $\psi_{h}$ could pose problems in numerical solvers that require evaluation of $\psi(T)$ in $T \in \mathbb{R}$. Additionally, vertical climb or descent yields induced velocities $\psi_{i}$ given by [29]

$$
\frac{\psi_{i}}{\psi_{h}}=-\frac{v_{c}}{\psi_{h}} \pm \sqrt{\left(\frac{v_{c}}{\psi_{h}}\right)^{2} \pm 1}
$$

where $v_{c}$ denotes the vertical climb velocity, and the sign ambiguity is dependent on whether the vehicle is in descent or climb. Notice that vehicle movement adds an additional complexity of sign change. Furthermore, $v_{c} / \psi_{h}>-1$ yields a turbulent wake state that precludes momentum theory employment; not even an extrapolated estimate is possible due to the domain of definition of Eq. (71). Finally, extraction of induced angles of attack and sideslip from Eq. (70) for use in $\pi$ theory yields convoluted algebra that precludes most optimization solvers, as illustrated in the following.

\section{A. $\pi$-Theory Wing-Propeller Interaction}

Traditional [28] aerodynamic forces and moments computation on a wing-propeller interaction scenario requires propwash-induced angle of attack $\alpha_{i}$ and sideslip $\beta_{i}$ obtained from $\boldsymbol{\psi}_{b}$ by solving Eq. (70) in the body reference frame. For this purpose, Eq. (70) is rewritten as

$$
\psi\left(\begin{array}{l}
\psi_{b 1} \\
\psi_{b 2} \\
\psi_{b 3}
\end{array}\right)=v_{\infty}^{2}\left(\begin{array}{c}
\cos \alpha \cos \beta \\
\sin \beta \\
\sin \alpha \cos \beta
\end{array}\right)+\left(\begin{array}{c}
\frac{T}{\rho S} \\
0 \\
0
\end{array}\right)
$$

Recall that, by definition, $\boldsymbol{v}_{\infty, b}$ freestream $\alpha$ and $\beta$ are given by

$$
\alpha=\tan ^{-1}\left(\frac{v_{\infty, b 3}}{v_{\infty, b 1}}\right)
$$

and

$$
\beta=\sin ^{-1}\left(\frac{v_{\infty, b 2}}{v_{\infty}}\right)
$$

Accordingly, propwash-induced $\alpha_{i}$ and $\beta_{i}$ are given as a function of $\psi$ by

$$
\alpha_{i}=\tan ^{-1}\left(\frac{\psi_{b 3}}{\psi_{b 1}}\right)
$$

and

$$
\beta_{i}=\sin ^{-1}\left(\frac{\psi_{b 2}}{\psi}\right)
$$

Substitution of Eq. (2) into Eqs. (다) and (다) yields

$$
\alpha_{i}=\tan ^{-1}\left(\frac{v_{\infty}^{2} \sin \alpha \cos \beta}{v_{\infty}^{2} \cos \alpha \cos \beta+(T / \rho S)}\right)
$$

and

$$
\beta_{i}=\sin ^{-1}\left(\frac{v_{\infty}^{2} \sin \beta}{\sqrt[4]{v_{\infty}^{4}+2 v_{\infty}^{2} \cos \alpha \cos \beta(T / \rho S)+(T / \rho S)^{2}}}\right)
$$

where $\alpha$ and $\beta$ are given by Eqs. (73) and (74). Notice that, in view of

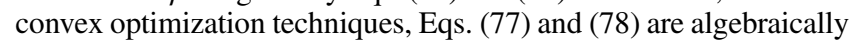
fairly complex.

\section{B. $\phi$-Theory Wing-Propeller Interaction}

The aforementioned numerical issues make traditional formulation impracticable for global TBV modeling. On the other hand, $\phi$ theory requires only $\psi \psi \psi$ for computation of static aerodynamic forces and moments, which are readily available in Eq. (70). For instance, static $\left(\boldsymbol{\omega}_{b}=\mathbf{0}\right)$ aerodynamic forces in the presence of propwash can be modeled as

$$
\boldsymbol{F}_{b}=-\frac{1}{2} \rho S \psi \Phi^{(f v)} \boldsymbol{\psi}=\underbrace{-\frac{1}{2} \rho S v_{\infty} \Phi^{(f v)} \boldsymbol{v}_{\infty}}_{\boldsymbol{F}_{b}^{(\infty)}}-\underbrace{\frac{S}{2 S_{p}} \Phi^{(f v)} \boldsymbol{T}_{b}}_{\boldsymbol{F}_{b}^{(p)}}
$$

In sharp contrast with $\pi$ theory, Eq. (79) is algebraically simple, bypasses $\alpha$ and $\beta$, and is defined for all $T \in \mathbb{R}$ (in fact, for all $\boldsymbol{T}_{b} \in \mathbb{R}^{3}$, if nonaxial propulsion forces are modeled in view of nonaxial flow). Additionally, under the presented physical assumptions, Eq. (79) sheds light on the structural composition of $\boldsymbol{F}_{b}$. Notice that $\boldsymbol{F}_{b}$ is composed of a superposition of an aerodynamic force $\boldsymbol{F}_{b}^{(\infty)}$ due to the freestream plus an independent contribution of $\boldsymbol{F}_{b}^{(p)}$ due to $\boldsymbol{T}_{b}$.

\section{Tilt-Body Physical and Mathematical Model}

The present section revisits central issues in TBV aerodynamics and propulsion modeling from the point of view of automatic control design and simulation. In parallel, the $\phi$-theory modeling approach is illustrated. The model aims toward numerical stability and simplicity, allowing for insightful controller synthesis while capturing dominant dynamical features (e.g., propeller gyroscopic effects, propellerwing interaction, and poststall phenomena). Notice that, although the scope of this work is TBVs, $\phi$ theory can be potentially applied at a larger class of HAVs.

\section{A. Overview of Qualitative Mechanics}

Figure 5 illustrates a typical TBV and its respective actuator inputs. In hover mode, notice that differential propeller speed (i.e., $\left.\left|\omega_{1}\right| \neq\left|\omega_{2}\right|\right)$ produces a $\hat{\boldsymbol{b}}_{1}$ moment by means of propeller reaction torques $\boldsymbol{N}_{i}$ in the fuselage and $\hat{\boldsymbol{b}}_{3}$ moments due to differential thrust forces (i.e., $\boldsymbol{T}_{1} \neq \boldsymbol{T}_{2}$ ). Additional $\hat{\boldsymbol{b}}_{1}$ moments are generated by means of differential elevon deflection $\delta_{i}$. Notice that such a moment is only apparent due to the propeller slipstream aerodynamic interaction with the elevons. Therefore, propeller-wing interaction modeling is of utmost importance to TBV control design. Then, $\hat{\boldsymbol{b}}_{2}$ moments are produced by means of symmetrical elevon deflections.

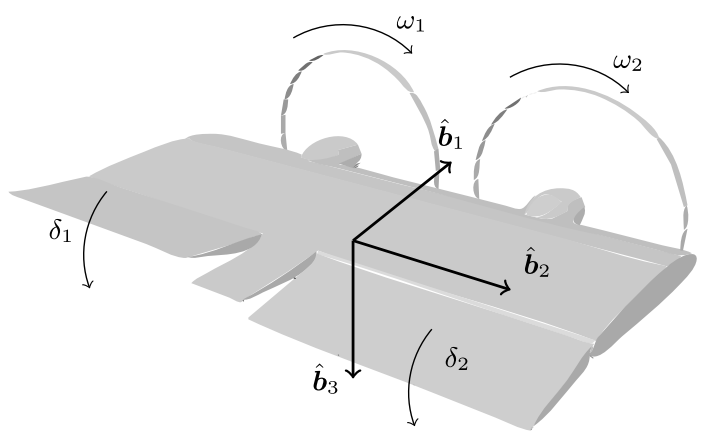

Fig. 5 Perspective view, body-axis definition, and actuation inputs for a typical tilt-body vehicle. 
Finally, velocity control is achieved by setting appropriate thrust intensity and TBV attitude.

On the other hand, in cruise flight, attitude control fundamentals remain the same but with increased aerodynamic efficiencies due to the additional air freestream velocity. Velocity control, however, becomes significantly more complex due to drag and lift forces that strongly depend on both velocity and attitude. Therefore, in cruise and transition flight regimes, the mathematical model shall prove its usefulness.

\section{B. Wet-Dry Wing Abstraction}

During transition flight, TBV aerodynamic surfaces experience high freestream velocity while maintaining considerable propeller speed. While doing so in high angles of attack, this notably creates two separate airflow regions on the wing (see Fig. 6).

One section is denominated the dry section and consists of the region of the flow over the wing unaffected by the propeller slipstream. In this region, the flow is reasonably assumed to behave aerodynamically similarly to the airfoil depicted in Fig. 4.

On the other hand, the second section is denominated the wet section and is composed of the wing region immersed in the propeller slipstream. In this region, the flow is assumed to be a superposition of a freestream flow due to relative wind-vehicle movement plus an induced propeller flow, as depicted in Fig. 7. The resulting aerodynamics are therefore computed by means of original airfoil coefficients applied at an induced (virtual) freestream $\psi$ according to Eq. (79).

\section{System of Rigid Bodies Model}

In the following, the six-degree-of-freedom equations of motion are applied at each of the three components (two propellers and wing) to obtain the complete dynamical behavior in view of general forces and moments. At first, consider the state equation for angular velocity

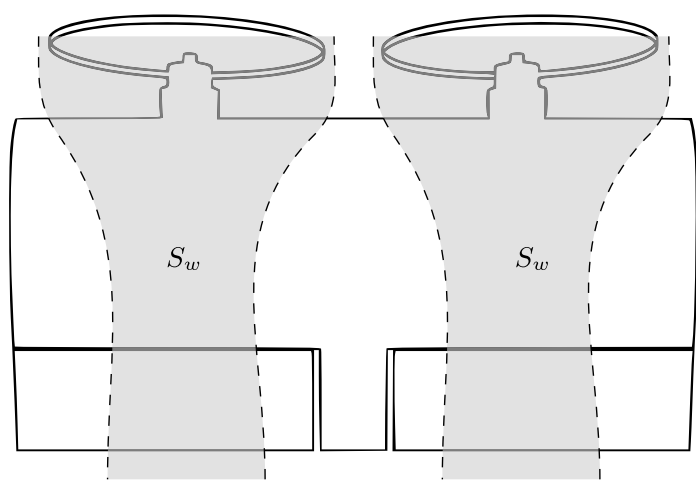

Fig. 6 Propeller-wing interaction: propeller wake slipstream illustration and the division between dry and wet sections. for a rigid body applied at a propeller $i(i \in\{1,2\})$ and described in the propeller $P_{i}$ coordinate system:

$$
\left.\left(\boldsymbol{N}_{i}-\boldsymbol{\tau}_{i}\right)_{p i}=J_{p} \frac{\mathrm{d}}{\mathrm{d} t}{ }^{l} \boldsymbol{\omega}_{p i}^{p i}+{ }^{l} \boldsymbol{\omega}_{p i}^{p i} \times\right] J_{p}{ }^{l} \boldsymbol{\omega}_{p i}^{p i}
$$

where $J_{p} \in \mathbb{R}^{3 \times 3}$ and ${ }^{l} \boldsymbol{\omega}_{p i}^{p i} \in \mathbb{R}^{3}$ denote, respectively, the propeller inertia matrix and propeller $P_{i}$ angular velocity with respect to the local north-east-down (NED) frame described in propeller $P_{i}$ coordinates. Hereafter, it is assumed that

$$
\left|J_{p} \frac{\mathrm{d}}{\mathrm{d} t}{ }^{l} \boldsymbol{\omega}_{p i}^{p i}\right| \ll\left|\left[{ }^{l} \boldsymbol{\omega}_{p i}^{p i} \times\right] J_{p}{ }^{l} \boldsymbol{\omega}_{p i}^{p i}\right|
$$

such that Eq. ( $\underline{80)}$ is simplified to read

$$
\left(\boldsymbol{\tau}_{i}\right)_{p i}=\left(\boldsymbol{N}_{i}\right)_{p i}-\left[{ }^{l} \boldsymbol{\omega}_{p i}^{p i} \times\right] J_{p}{ }^{l} \boldsymbol{\omega}_{p i}^{p i}
$$

Equation (82) relates the internal torque $\boldsymbol{\tau}_{i}$ to the propulsion moment $\boldsymbol{N}_{i}$ and the gyroscopic effect given by $\left.{ }^{l} \boldsymbol{\omega}_{p i}^{p i} \times\right] J_{p}{ }^{l} \boldsymbol{\omega}_{p i}^{p i}$. By means of the additivity property of angular velocities,

$$
{ }^{l} \boldsymbol{\omega}_{p i}^{p i}={ }^{l} \boldsymbol{\omega}_{p i}^{b}+{ }^{b} \boldsymbol{\omega}_{p i}^{p i}=D_{p i}^{b l} \boldsymbol{\omega}_{b}^{b}+\left(\begin{array}{c}
\omega_{i} \\
0 \\
0
\end{array}\right)
$$

Notice that the direction cosine matrix $D_{p i}^{b}$ represents a simple rotation of $\theta_{i}$ with respect to the $\hat{\boldsymbol{b}}_{1}$ axis and can be written as

$$
D_{p i}^{b}\left(\theta_{i}\right)=\left[\begin{array}{cc}
1 & \mathbf{0}_{1 \times 2} \\
\mathbf{0}_{2 \times 1} & C_{p i}^{b}\left(\theta_{i}\right)
\end{array}\right], \quad C_{p i}^{b}\left(\theta_{i}\right)=\left[\begin{array}{cc}
\cos \theta_{i} & \sin \theta_{i} \\
-\sin \theta_{i} & \cos \theta_{i}
\end{array}\right]
$$

Accordingly, each of the gyroscopic factors are rewritten as

$$
\begin{gathered}
\boldsymbol{\omega}_{p i}^{p i}=\left[\begin{array}{cc}
1 & \mathbf{0}_{1 \times 2} \\
\mathbf{0}_{2 \times 1} & C_{p i}^{b}\left(\theta_{i}\right)
\end{array}\right]\left(\begin{array}{l}
P \\
Q \\
R
\end{array}\right)=\left(\begin{array}{c}
P+\omega_{i} \\
\left.C_{p i}^{b}\left(\begin{array}{l}
Q \\
R
\end{array}\right)\right)(85)
\end{array}\right) \\
{\left[^{l} \boldsymbol{\omega}_{p i}^{p i} \times\right]=\left[\begin{array}{cc}
Q & R) C_{b}^{p i}\left[\begin{array}{cc}
0 & 1 \\
-10
\end{array}\right] \\
{\left[\begin{array}{cc}
0 & 1 \\
-1 & 0
\end{array}\right] C_{p i}^{b}\left(\begin{array}{l}
Q \\
R
\end{array}\right)} & \left(P+\omega_{i}\right)\left[\begin{array}{ll}
0 & -1 \\
1 & 0
\end{array}\right]
\end{array}\right]}
\end{gathered}
$$

and

$$
J_{p}=\left[\begin{array}{ccc}
J_{p x} & 0 & 0 \\
0 & J_{p n} & 0 \\
0 & 0 & J_{p n}
\end{array}\right]
$$

Notice that $J_{p}$ is modeled as a disk, hence its diagonal shape. Performing the gyroscopic product yields

$$
\left(\boldsymbol{\tau}_{i}\right)_{b}=\left(N_{i}\right)_{b}-\left(P+\omega_{i}\right)\left(J_{p x}-J_{p n}\right)\left(\begin{array}{c}
0 \\
R \\
-Q
\end{array}\right)
$$

after an appropriate basis change. Subsequently, consider the state equation for angular velocity for a rigid body applied at the fuselage and described in the fuselage $B$ coordinate system:
Fig. 7 Resulting aerodynamics (namely, induced relative air velocity $\psi$ with induced angle of attack $\alpha_{i}$ ) as a superposition of freestream $v_{\infty}$ and induced propeller wake $v_{i}(T)$. 


$$
\begin{aligned}
& \left(\sum_{i=1}^{2}\left(\boldsymbol{M}_{i}+\boldsymbol{\tau}_{i}+\boldsymbol{r}^{p i / b} \times \boldsymbol{T}_{i}+\boldsymbol{r}^{a i / b} \times \boldsymbol{A}_{i}\right)\right)_{b} \\
& =J_{b} \frac{\mathrm{d}}{\mathrm{d} t} l \boldsymbol{\omega}_{b}^{b}+\left[{ }^{l} \boldsymbol{\omega}_{b}^{b} \times\right] J_{b}^{l} \boldsymbol{\omega}_{b}^{b}
\end{aligned}
$$

where $\boldsymbol{A}_{i}$ and $\boldsymbol{T}_{i}$ are, respectively, the aerodynamic forces in the $i$ wing and propeller thrust. Finally, ${ }^{l} \boldsymbol{\omega}_{b}^{b}$ is used to update the quaternion attitude $\boldsymbol{q} \in \mathbb{R}^{4}$ according to

$$
\frac{\mathrm{d} \boldsymbol{q}}{\mathrm{d} t}=\frac{1}{2}\left[\begin{array}{cc}
0 & -\left({ }^{l} \boldsymbol{\omega}_{b}^{b}\right)^{T} \\
{ }^{l} \boldsymbol{\omega}_{b}^{b} & -\left[{ }^{l} \boldsymbol{\omega}_{b}^{b} \times\right]
\end{array}\right] \boldsymbol{q}
$$

The translation counterpart of the foregoing development is given by Newton's second law of motion applied at the fuselage, yielding

$$
m \boldsymbol{g}_{l}+D_{l}^{b} \sum_{i=1}^{2}\left(\boldsymbol{T}_{i}+\boldsymbol{A}_{i}\right)=m \frac{\mathrm{d}}{\mathrm{d} t} l^{l} \boldsymbol{v}_{b}^{b}
$$

where $m, \boldsymbol{g}_{l}$, and ${ }^{l} \boldsymbol{\omega}_{l}^{b}$ denote, respectively, the vehicle total mass, the gravity in NED coordinates, and the inertial velocity of the vehicle in NED coordinates. Furthermore,

$$
\frac{\mathrm{d}}{\mathrm{d} t} \boldsymbol{p}_{l}^{b}={ }^{l} \boldsymbol{v}_{b}^{b}
$$

\section{Aerodynamics and Propulsion Wrap-Up}

The propeller's thrust $\boldsymbol{T}_{i}$ and moment $\boldsymbol{N}_{i}$ are modeled according to the widely used relations

$$
\boldsymbol{T}_{i}=k_{f} \omega_{i}^{2} \hat{\boldsymbol{b}}_{1} \quad k_{f}>0
$$

and

$$
\boldsymbol{N}_{i}=-\operatorname{sign}\left(\omega_{i}\right) k_{m} \omega_{i}^{2} \hat{\boldsymbol{b}}_{1} \quad k_{m}>0
$$

The direction of each propeller rotation is chosen so as to coincide with the induced banking motion due to differential lift and differential propeller moments. This is achieved by imposing $\omega_{1}<0$ and $\omega_{2}>0$ (recall the $\omega_{i}$ sign conventions in Fig. 5).

Aerodynamics are modeled according to the thin airfoil $\phi$ theory set forth in Sec. IV.C, with the additional elevon deflection modeled as a cambered airfoil of variable camber according to

$$
\Phi^{(f v)}\left(\delta_{i}\right)=\Phi_{0}^{(f v)}\left(I-\delta_{i}\left[\zeta_{f} \times\right]\right)
$$

To understand the motivation behind Eq. (95), recall from Sec. IV.C that cambered airfoils are modeled by means of $\boldsymbol{a}_{\phi 1}$ and $\boldsymbol{a}_{\phi 2}$ rotation, and that small-angle rotations $\zeta=\left(\zeta_{1}, \zeta_{2}, \zeta_{3}\right)$ are approximately described by

$$
D(\zeta) \approx I-[\zeta \times] \text { for small } \zeta
$$

where $\zeta \in \mathbb{R}^{3}$ is hereafter termed elevon effectiveness. A similar development is carried out for moment coefficients. Finally, taken together, this section's assumptions lead to the TBV force and moment breakdown given by Eqs. (97) and (98), as well as the TBV equations of motion defined in the following. Although lengthy, the overall force and moment expressions are algebraically simple, singularity free, and indeed bypass all occurrences of $\alpha$ and $\beta$ (including propwash induced):

$$
\begin{aligned}
\sum \boldsymbol{F}_{b} & =\boldsymbol{T}_{1}+\boldsymbol{T}_{2}-\frac{S}{4 S_{p}} \boldsymbol{\Phi}^{(f v)} \boldsymbol{T}_{1}-\frac{S}{4 S_{p}} \boldsymbol{\Phi}^{(f v)} \boldsymbol{T}_{2}-\frac{1}{2} \rho S \Phi^{(f v)} v \boldsymbol{v}_{b} \\
& +m D \boldsymbol{g}_{l}+\frac{1}{4} \rho S \Phi^{(f v)}\left[\boldsymbol{\zeta}_{f} \times\right]\left(\delta_{1}+\delta_{2}\right) v \boldsymbol{v}_{b}-\frac{1}{2} \rho S \Phi^{(m v)} v B \boldsymbol{\omega}_{b} \\
& +\frac{S}{4 S_{p}} \Phi^{(f v)}\left[\boldsymbol{\zeta}_{f} \times\right] \delta_{1} \boldsymbol{T}_{1}+\frac{S}{4 S_{p}} \Phi^{(f v)}\left[\boldsymbol{\zeta}_{f} \times\right] \delta_{2} \boldsymbol{T}_{2} \\
& +\frac{1}{4} \rho S \Phi^{(m v)}\left[\zeta_{f} \times\right]\left(\delta_{1}+\delta_{2}\right) B v \boldsymbol{\omega}_{b}
\end{aligned}
$$

$$
\begin{aligned}
& \sum \boldsymbol{M}_{b}=-\frac{1}{2} \rho S B \Phi^{(m v)} v \boldsymbol{v}_{b}-\frac{1}{2} \rho S B \Phi^{(m \omega)} v B \boldsymbol{\omega}_{b}-\frac{S}{4 S_{p}} B \Phi^{(m v)} \boldsymbol{T}_{1} \\
& -\frac{S}{4 S_{p}} B \Phi^{(m v)} \boldsymbol{T}_{2}+\left[\boldsymbol{p}_{1} \times\right] \boldsymbol{T}_{1}+\left[\boldsymbol{p}_{2} \times\right] \boldsymbol{T}_{2}+ \\
& \quad-\frac{S}{4 S_{p}}\left[\boldsymbol{a}_{1} \times\right] \Phi^{(f v)} \boldsymbol{T}_{1}-\frac{S}{4 S_{p}}\left[\boldsymbol{a}_{2} \times\right] \Phi^{(f v)} \boldsymbol{T}_{2} \\
& +\frac{S}{4 S_{p}}\left[\boldsymbol{a}_{1} \times\right] \Phi^{(f v)}\left[\boldsymbol{\zeta}_{f} \times\right] \delta_{1} \boldsymbol{T}_{1}+\frac{S}{4 S_{p}}\left[\boldsymbol{a}_{2} \times\right] \Phi^{(f v)}\left[\boldsymbol{\zeta}_{f} \times\right] \delta_{2} \boldsymbol{T}_{2} \\
& +\boldsymbol{N}_{1}+\boldsymbol{N}_{2}+\frac{1}{4} \rho S\left[\boldsymbol{a}_{1} \times\right] \Phi^{(f v)}\left[\boldsymbol{\zeta}_{f} \times\right] \delta_{1} v \boldsymbol{v}_{b} \\
& +\frac{1}{4} \rho S\left[\boldsymbol{a}_{2} \times\right] \Phi^{(f v)}\left[\boldsymbol{\zeta}_{f} \times\right] \delta_{2} v \boldsymbol{v}_{b}+\frac{1}{4} \rho S\left[\boldsymbol{a}_{1} \times\right] \Phi^{(m v)}\left[\boldsymbol{\zeta}_{f} \times\right] \delta_{1} B v \boldsymbol{\omega}_{b}+ \\
& +\frac{1}{4} \rho S\left[\boldsymbol{a}_{2} \times\right] \Phi^{(m v)}\left[\boldsymbol{\zeta}_{f} \times\right] \delta_{2} B v \boldsymbol{\omega}_{b}+\frac{1}{4} \rho S B \Phi^{(m v)}\left[\boldsymbol{\zeta}_{m} \times\right]\left(\delta_{1}+\delta_{2}\right) v \boldsymbol{v}_{b} \\
& +\frac{1}{4} \rho S B \Phi^{(m \omega)}\left[\boldsymbol{\zeta}_{m} \times\right]\left(\delta_{1}+\delta_{2}\right) v B \boldsymbol{\omega}_{b}+\frac{S}{4 S_{p}} B \Phi^{(m v)}\left[\boldsymbol{\zeta}_{m} \times\right] \delta_{1} \boldsymbol{T}_{1} \\
& +\frac{S}{4 S_{p}} B \Phi^{(m v)}\left[\boldsymbol{\zeta}_{m} \times\right] \delta_{2} \boldsymbol{T}_{2}
\end{aligned}
$$

Definition VI.1 (tilt-body vehicle system): Let $B$ be a tilt-body vehicle with inertia $\left(m, J_{b}, J_{p}\right)$. A tilt-body vehicle system (TBVS) is defined as the associated dynamical system given by

$$
\left\{\begin{array}{l}
\dot{\boldsymbol{v}}_{b}=\frac{1}{m} \sum \boldsymbol{F}_{b}(\boldsymbol{x}, \boldsymbol{u}, \boldsymbol{w})-\left[\boldsymbol{\omega}_{b} \times\right] \boldsymbol{v}_{b} \\
\dot{\boldsymbol{\omega}}_{b}=J^{-1} \sum \boldsymbol{M}_{b}(\boldsymbol{x}, \boldsymbol{u}, \boldsymbol{w})-J^{-1}\left[\boldsymbol{\omega}_{b} \times\right] J \boldsymbol{\omega}_{b} \\
\dot{D}_{b}^{l}=-\left[\boldsymbol{\omega}_{b} \times\right] D_{b}^{l}
\end{array}\right.
$$

where

$$
\sum \boldsymbol{F}_{b}(\boldsymbol{x}, \boldsymbol{u}, \boldsymbol{w})
$$

and

$$
\sum M_{b}(x, u, w)
$$

are given by Eqs. (97) and (98). Additionally, $x$ and $u$ are defined as $\boldsymbol{x}=\left(\boldsymbol{v}_{b}, \boldsymbol{\omega}, D_{b}^{l}\right)$ and $\boldsymbol{u}=\left(\overline{\omega_{1}}, \omega_{2}, \delta_{1}, \delta_{2}\right)$, whereas $\boldsymbol{w} \in \mathbb{R}^{3}$ denotes the external wind disturbance.

\section{Longitudinal Flight Analysis}

A longitudinal flight analysis is carried out by an equilibrium point analysis (existence, uniqueness, and computation results). For the present longitudinal analysis, no external wind (without loss of generality) is assumed; thus, the six-degree-of-freedom TBV dynamical model simplification motivates the following definition.

Definition VII.1 (longitudinal tilt-body system): Let $B$ be a TBVS with inertia $\left(m, J_{b}, J_{p}\right)$ such that 1) $\omega_{2}(t)=-\omega_{1}(t)=\omega(t)$; 2) $\left.\delta_{2}(t)=\delta_{1}(t)=\delta(t) ; 3\right)$ 


$$
D_{b}^{l}(0)=\left[\begin{array}{ccc}
\cos \theta(0) & 0 & -\sin \theta(0) \\
0 & 1 & 0 \\
\sin \theta(0) & 0 & \cos \theta(0)
\end{array}\right]
$$

4) $\boldsymbol{v}_{b}(0)=\left(\begin{array}{llll}v_{b 1}(0) & 0 & v_{b 3}(0)\end{array}\right)^{T}$, and 5) $\boldsymbol{\omega}_{b}(0)=\left(\begin{array}{lll}0 & \dot{\theta}(0) & 0\end{array}\right)^{T}$.

The longitudinal tilt-body system is defined as the simplified dynamical system given by

$$
\left\{\begin{array}{l}
\left(\begin{array}{l}
\dot{v}_{b 1} \\
\dot{v}_{b 3}
\end{array}\right)=\left[\begin{array}{cc}
0 & 1 \\
-1 & 0
\end{array}\right] \dot{\theta} \boldsymbol{v}_{b}+1 m \sum \boldsymbol{F}_{b}(\boldsymbol{x}, \boldsymbol{u}) \\
\ddot{\theta}=J_{y y}^{-1} \sum M_{b 2}(\boldsymbol{x}, \boldsymbol{u})
\end{array}\right.
$$

where for the remainder of this section, $x \in \mathbb{R}^{4}$ such that $\boldsymbol{x}=\left(v_{b 1}, v_{b 3}, \theta, \dot{\theta}\right), \boldsymbol{u} \in \mathbb{R}^{2}$ such that $\boldsymbol{u}=(\omega, \delta)$, and $\theta$ denotes the pitch angle.

\section{A. Equilibrium Points}

The following subset of equilibrium points of Eq. (100) are of interest in view of the nominal flight conditions.

Definition VII.2 (cruise points): A point $(\boldsymbol{x}, \boldsymbol{u}) \in \mathbb{R}^{4} \times \mathbb{R}^{2}$ is called a cruise equilibrium point if 1 )

$$
\sum \boldsymbol{F}_{b}(\boldsymbol{x}, \boldsymbol{u})=\sum \boldsymbol{M}_{b}(\boldsymbol{x}, \boldsymbol{u})=\mathbf{0}
$$

(mechanical equilibrium condition) 2) $v_{l 3}=0$ (level flight condition); and 3) $\dot{\theta}=0$ (zero pullup condition).

Furthermore, the set of all cruise equilibrium points is denoted as $\Theta_{0} \subset \mathbb{R}^{6}$.

Therefore, $(\boldsymbol{x}, \boldsymbol{u}) \in \Theta_{0}$ if, and only if,

$$
\left\{\begin{array}{c}
\sum \boldsymbol{F}_{b}=\mathbf{0} \\
\sum M_{b 2}=0
\end{array}\right.
$$

which can be rewritten as

$$
M(\theta)\left(\begin{array}{c}
v_{l 1}^{2} \\
\delta v_{l 1}^{2} \\
\delta T \\
T
\end{array}\right)=\underbrace{m g\left(\begin{array}{c}
s \theta \\
-c \theta \\
0
\end{array}\right)}_{\boldsymbol{b}(\theta)}
$$

where

$$
M(\theta)=\left[\begin{array}{cc}
-\frac{1}{2} \rho S \Phi^{(f v)}\left(\begin{array}{c}
c \theta \\
s \theta
\end{array}\right) & \frac{1}{2} \rho S \Phi^{(f v)}\left[\zeta_{f} \times\right]\left(\begin{array}{c}
c \theta \\
s \theta
\end{array}\right) \\
-\frac{1}{2} \rho S c \Phi^{(m v)}\left(\begin{array}{c}
c \theta \\
s \theta
\end{array}\right) & \frac{c}{2} \rho S \Phi^{(m v)}\left[\zeta_{m} \times\right]\left(\begin{array}{c}
c \theta \\
s \theta
\end{array}\right)
\end{array}\right.
$$

Lemma VII.1: If $\xi_{m}, \xi_{f} \in \mathbb{R}^{*}$ and $\xi_{m} \neq \xi_{f}$, then $M(\theta)$ is full rank for all $\theta \in \mathbb{R} \backslash \mathbb{I}(\Phi)$.

Proof: Consider the submatrix $M^{\prime}(\theta) \in \mathbb{R}^{3 \times 3}$ of $M(\theta)$ consisting of the first, second, and third columns, i.e.,

$$
M^{\prime}(\theta)=M(\theta)\left[\begin{array}{lll}
1 & 0 & 0 \\
0 & 1 & 0 \\
0 & 0 & 1 \\
0 & 0 & 0
\end{array}\right]
$$

Algebraic manipulation of $\operatorname{det}\left(M^{\prime}(\theta)\right)$ yields

$$
\operatorname{det}\left(M^{\prime}(\theta)\right)=\frac{1}{8} \rho^{2} c \frac{S^{3}}{S_{p}}\left(\zeta_{f}-\zeta_{m}\right) \zeta_{f} \operatorname{det} \Phi^{(f v)} s \theta \Phi^{(m v)}\left(\begin{array}{c}
c \theta \\
s \theta
\end{array}\right)
$$

Notice that $\Phi^{(f v)}>0$, thus $\operatorname{det} \Phi^{(f v)} \neq 0$. Additionally, $\sin \theta \neq 0$ and $\Phi^{(m v)}(c \theta \quad s \theta)^{T} \neq 0$ because $\theta \notin \mathbb{I}(\Phi)$. Therefore, $\operatorname{det}\left(M^{\prime}(\theta)\right) \neq 0$ and $\operatorname{rank}\left(M^{\prime}(\theta)\right)=3$. However, because $M^{\prime}(\theta)$ is a submatrix of $M(\theta)$, then $\operatorname{rank}(M(\theta))=3$ and $M(\theta)$ is full rank.

Corollary VII.1: If $\xi_{m}, \xi_{f} \in \mathbb{R}^{*}$ and $\xi_{m} \neq \xi_{f}$, and $\Phi$ is of the thinairfoil class, then $M(\theta)$ is full rank for all $\theta \neq k \pi, k \in \mathbb{Z}$.

Lemma VII.1 implies that, except for $\theta \in \mathbb{I}(\Phi)$, the reduced row echelon form of $M(\theta) \boldsymbol{\xi}=\boldsymbol{b}(\theta)$ is of the form

$$
\left[\begin{array}{ll}
M(\theta) & \boldsymbol{b}(\theta)
\end{array}\right] \sim\left[\begin{array}{ccccc}
1 & 0 & 0 & -v_{1} & b_{1} \\
0 & 1 & 0 & -v_{2} & b_{2} \\
0 & 0 & 1 & -v_{3} & b_{3}
\end{array}\right]
$$

and thus

$$
\boldsymbol{\xi}=\left(\begin{array}{llll}
b_{1} & b_{2} & b_{3} & 0
\end{array}\right)^{T}+\epsilon\left(\begin{array}{llll}
v_{1} & v_{2} & v_{3} & 1
\end{array}\right)^{T}, \quad \epsilon \in \mathbb{R}
$$

Substitution of Eq. (109) in the second condition of Eq. (104) yields

$$
\left.\begin{array}{cc}
\frac{1}{2} \frac{S}{S_{p}} \Phi^{(f v)}\left[\zeta_{f} \times\right]\left(\begin{array}{l}
1 \\
0
\end{array}\right) & \left(\begin{array}{l}
2 \\
0
\end{array}\right) \frac{1}{2} \frac{S}{S_{p}} \Phi^{(f v)}\left(\begin{array}{l}
1 \\
0
\end{array}\right) \\
\frac{c}{2} \frac{S}{S_{p}} \Phi^{(m v)}\left[\zeta_{m} \times\right]\left(\begin{array}{l}
1 \\
0
\end{array}\right) & -\frac{c}{2} \frac{S}{S_{p}} \Phi^{(m v)}\left(\begin{array}{l}
1 \\
0
\end{array}\right)
\end{array}\right]
$$

Notice that, without loss of generality, a zero dry propwash area is assumed, i.e., $S_{d}=0$. Furthermore, for a given $\theta$, Eq. (102) is decomposed into a linear system and a simpler set of nonlinear equations such that

$$
\left\{\begin{array}{cc}
M(\theta) \boldsymbol{\xi}=\boldsymbol{b}(\theta), & \boldsymbol{\xi} \in \mathbb{R}^{4} \\
\left(\xi_{2}, \xi_{3}\right)=\delta\left(\xi_{1}, \xi_{4}\right), & \delta \in \mathbb{R}
\end{array}\right.
$$

The dimension of the affine linear space $M(\theta) \boldsymbol{\xi}=\boldsymbol{b}(\theta)$ is dependent on $\operatorname{rank}(M(\theta))$. The following lemma is the starting point of $\Theta_{0}$ computation.

Definition VII.3 ( $\delta$-inefficient angles): The set $\mathbb{I}(\Phi) \subset \mathbb{R}$ of $\delta$-inefficient angles is defined as

$$
\left\{\begin{array}{l}
b_{2}+\epsilon v_{2}=\delta\left(b_{1}+\epsilon v_{1}\right) \\
b_{3}+\epsilon v_{3}=\delta \epsilon
\end{array}\right.
$$

which, by solving for $\epsilon$, yields the following thrust characteristic polynomial $p_{t}(\epsilon)$ (because its roots yield potential cruise $T$ values)

$$
\left(v_{2}-v_{1} v_{3}\right) \epsilon^{2}+\left(b_{2}-b_{1} v_{3}-b_{3} v_{1}\right) \epsilon-b_{1} b_{3}=0
$$

Notice that Eq. (111) allows for, at most, two real solutions and upper bounds the number of possible cruise states at any given $\theta$. Backsubstitution of the roots of $p_{t}(\epsilon)$ in Eq. (109) yields the cruise points for a given $\theta$. The following results highlight these notions. 
Theorem VII.1 ( $\phi$-trim algorithm): For a given $\theta \in \mathbb{R} \backslash \mathbb{I}(\Phi)$, if $p_{t}(\epsilon)$ has no real roots, then $\Theta_{0}(\theta)=\varnothing$. Otherwise, let $\epsilon_{1}$ and $\epsilon_{2}$ be its real roots. Thus,

$$
\left(\sqrt{\xi_{1}\left(\epsilon_{i}\right)}, 0,0, \theta, \sqrt{\frac{\epsilon_{i}}{k_{f}}}, \frac{\xi_{3}\left(\epsilon_{i}\right)}{\epsilon_{i}}\right) \in \Theta_{0}(\theta)
$$

if, and only if, $\epsilon_{i}>0$ and $\xi_{1}\left(\epsilon_{i}\right) \geq 0$.

Corollary VII.2 (on the number of cruise points): For a given $\theta \in \mathbb{R} \backslash \mathbb{I}(\Phi)$, there are, at most, two distinct $(\boldsymbol{x}, \boldsymbol{u}) \in \Theta_{0}$ such that $x_{4}=\theta$.

Notice that Theorem VII.1 offers the possibility of computationally checking for the existence of cruise points as well as their accurate, guaranteed, and fast computation. Notice that no initial guesses nor algorithm iterations are required. Theorem VII.1 is a powerful tool for online planning algorithms [23] that requires fast online computation of equilibrium points, and it provides a bulletproof algorithm (in comparison to general nonlinear root solver algorithms, such as genetic algorithms and Newton-Raphson) that outperforms other solutions.

\section{B. Wind-Tunnel Experiments}

No matter how elegant, a mathematical model is of little practical use if not coherent with reality. Taken together, Secs. VI.D and VII.A provide a powerful algorithm to theoretically compute preliminary operating conditions for longitudinal flight, even (and especially) if wind-tunnel and flight data are unavailable. If consistent with reality, $\phi$ theory provides a model for supporting flight stabilization routine implementation and immediate test flights. This avoids wind-tunnel campaigns aimed at obtaining controls-related data and, ultimately, speeds up development. To check whether $\phi$ theory is consistent with reality, a wind-tunnel campaign is conducted. Hopefully, consistency leads to successful flights, which leads to less usage of wind-tunnel campaigns in the future for control designs (of course, wind-tunnel campaigns are still invaluable for aerodynamic design insights). The main objective of this section is therefore comparing trim points computed based on Sec. VII.A against real data from wind-tunnel measurements.

Notice that, although the wind-tunnel campaign targets static conditions, dynamic terms will apparently remain unvalidated. Nevertheless, an equivalence between $\phi$ and the well-established $\pi$-dynamic coefficients was derived in Sec. IV.B. This obviates the intricate dynamic wind-tunnel tests for dynamic $\phi$-coefficients validation.

The experiments were conducted at the low Reynolds SabRe closed-loop wind tunnel (Fig. 8) located at ISAE-SUPAERO and were capable of delivering low Reynolds stable and uniform flow at a velocity range of $2-25 \mathrm{~m} / \mathrm{s}$, which was thus ideal for experimenting with full-span micro air vehicles $(2.4 \times 1.2 \times 0.8 \mathrm{~m}$ test section). Forces and moments were measured by means of a calibrated fivecomponent internal balance.

An adapted TBV, namely, the MAVion, was manufactured for wind-tunnel campaign purposes. Its main objectives were to enable rigid installment of the internal balance in both configurations and to provide a nondeformable airfoil section for aerodynamic testing. It had a210 $\mathrm{mm}$ chord and a $420 \mathrm{~mm}$ wingspan. The elevons corresponded to $33 \%$ of its MH45 profile chord. Figure 9 illustrates

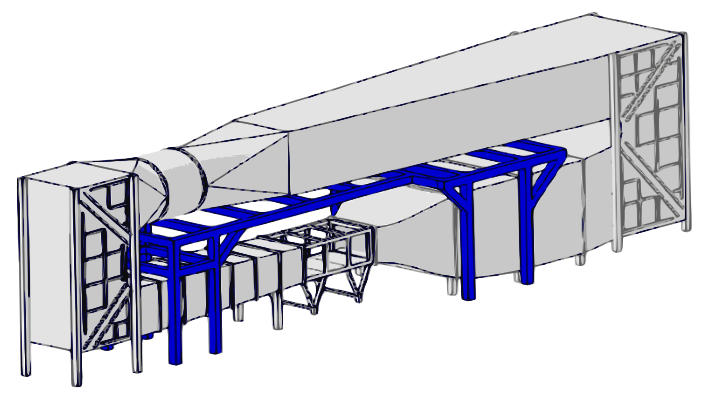

Fig. 8 SabRe closed-loop wind-tunnel facility. the electronic setup counterpart. Notice that elevon deflections were measured by means of potentiometers installed directly at the elevons, avoiding servo measurements that were susceptible to inaccuracy due to rod deformations and servo errors. On the other hand, propeller speeds were measured by the dc speed controllers. Finally, an Xbee radio module communicates telemetry and commands to a ground station (see Fig. 10) by means of an embedded ARM7-based microcontroller.

Because the test section is closed, large pitch angles may cause the flow to impinge upon the lower vane surface. Previous work [30] evaluated this effect and found that wall phenomena were unnoticeable up to a $60 \mathrm{deg}$ pitch. Pitch angles above this value introduced increased blockage and wall effects. Future work could identify corrections to dynamic pressure, lift, and drag. In the scope of the present project, future efforts will be toward obtaining data from indoor and outdoor flight experiments.

The dataset comprises the wind relative velocity, elevon deflection, and propeller rotation required to achieve static equilibrium in a given angle of attack. The fundamental idea of the experimental procedure is to, for each sampled $\alpha$, iteratively search for values $V_{\infty}, \delta_{i}$, and $\omega_{i}$ that will deliver aerodynamic/propulsion forces and moments (notice gravitational suppression) that will cancel gravitational forces and moments on the flying model, which has a different mass distribution than the wind-tunnel model (that is not meant to fly). The desired flying model mass distribution is such that the center of mass is longitudinally located at a position $0.15 c$ behind the leading edge, which is motivated by a $10 \%$ longitudinal static stability margin requirement. The results can be seen in Fig. 11.

The results validate the design by experimentally demonstrating that the MAVion is capable of sustaining flight from 0 to $20 \mathrm{~m} / \mathrm{s}$ in the absence of wind. Equivalently speaking, the MAVion is capable of maintaining hover flight in adverse wind conditions up to $20 \mathrm{~m} / \mathrm{s}$. It is noted that maximum speed is not reached in the wind tunnel due to internal balance strain gauge saturation. However, the steep rise in

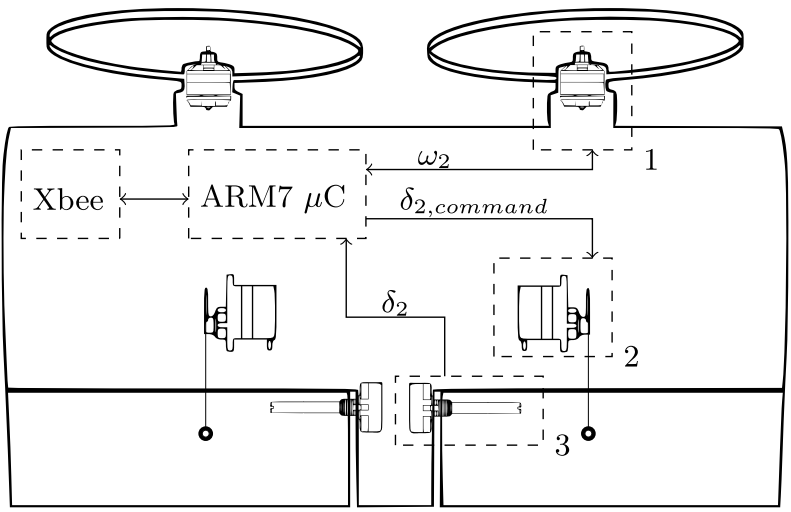

Fig. 9 TBV wind-tunnel model instrumentation.

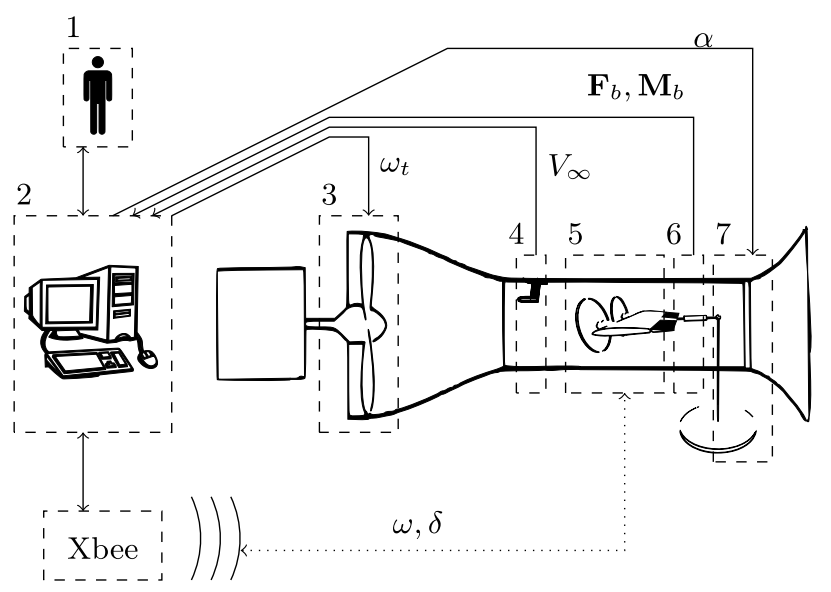

Fig. 10 Wind-tunnel acquisition system setup. 

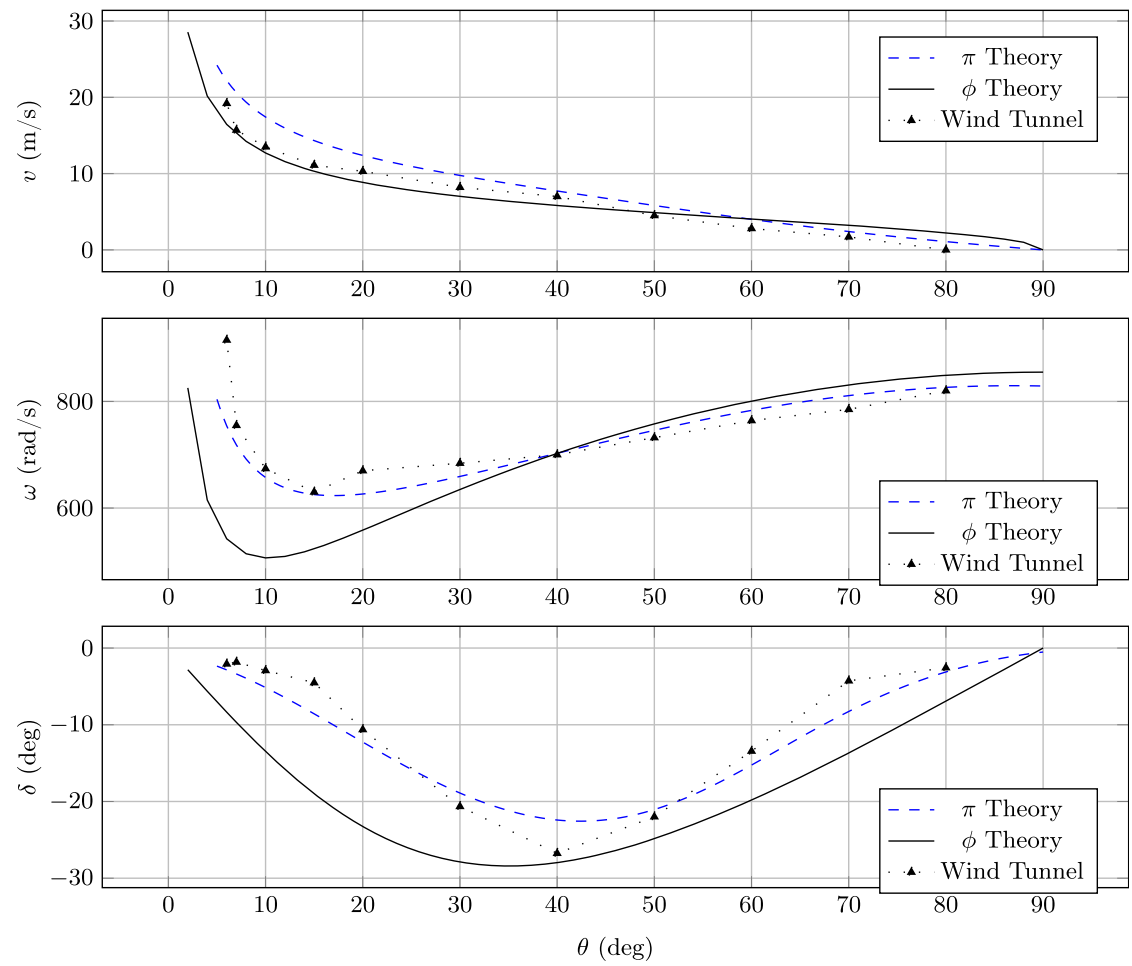

Fig. 11 Longitudinal cruise points for a given test vehicle.

propeller rotation speed $\omega$ for small $\theta$ in the equilibrium figures suggests an upper bound of $v_{n} \leq 20 \mathrm{~m} / \mathrm{s}$. Finally, the elevator deflection angle shows a maximum of $\delta=27 \mathrm{deg}$ at $\alpha=40 \mathrm{deg}$, which is within the range of the elevon aerodynamic efficiency. This confirms that the propeller slipstream is strong enough to guarantee pitch control throughout the entire transition flight envelope.

The $\pi$-theory results employ aerodynamic coefficients that are based on truncated Fourier series of order two to fit wind-tunnel data. Additionally, propeller force coefficients are compensated to account for oblique airflow. Notice that $\phi$ theory produces remarkably close results without resorting to wind-tunnel campaigns. On the other hand, $\pi$ theory provides a superior fit but requires experimental data. Additionally, relatively accurate initial guesses are necessary for convergence while numerically computing $\pi$-theory trim points using the optimization routine fsolve in MATLAB. In this study, $\phi$ theory provides initial conditions for the $\pi$-theory trim point solver, and successful convergence is achieved for all points of interest.

\section{Conclusions}

This paper outlines the fundamental ideas behind the newly proposed $\phi$ theory and its basic properties. It is believed that this work is the first attempt to study sufficient aerodynamic coefficients structure in view of facilitating 1) algorithmic control design and 2) qualitative behavior study of highly maneuverable aerial vehicles. Deliberately, $\phi$ theory does not pursue a more accurate description of reality. Indeed, $\phi$ theory is less accurate when compared to most other modeling approaches available in the literature. Instead, a minimal description that is adequate and sufficient for SOS-optimization-based guidance and control purposes (with reasonable robustness margins) is pursued. However, care is exercised to not oversimplify reality. This paper aims at finding an optimal tradeoff between complexity and usefulness (in view of control design), and wind-tunnel data to validate $\phi$ theory are provided.

Hopefully, $\phi$ theory lays the groundwork for substantially interesting opportunities in hybrid aerial vehicle (HAV) trajectory planning and algorithmic control, and it renders HAVs more accessible to the general control and robotics communities.

\section{References}

[1] Fay, C. B., "A Cursory Analysis of the VTOL Tilt-Wing Performance and Control Problems," Annals of the New York Academy of Sciences, Vol. 107, March 1963, pp. 102-146. doi:10.1111/j.1749-6632.1963.tb13275.x

[2] Girard, P. F., and Everett, W. L., "ATest Pilot Report on the X-13 Vertijet and VZ-3RY Vertiplane," Annals of the New York Academy of Sciences, Vol. 107, March 1963, pp. 409-427. doi:10.1111/j.1749-6632.1963.tb13289.x

[3] Schmidt, C. F., "Flight Safety in VTOL Aircraft," Annals of the New York Academy of Sciences, Vol. 107, March 1963, pp. 49-56. doi:10.1111/j.1749-6632.1963.tb13270.x

[4] Hartmann, P., Meyer, C., and Moorman, D., "Unified Velocity Control and Flight State Transition of Unmanned Tilt-Wing Aircraft," Journal of Guidance, Control, and Dynamics, Vol. 40, No. 6, 2017, pp. 1348-1359. doi:10.2514/1.G002168

[5] Sato, M., and Muraoka, K., "Flight Controller Design and Demonstration of Quad-Tilt-Wing Unmanned Aerial Vehicle," Journal of Guidance, Control, and Dynamics, Vol. 38, No. 6, 2015, pp. 1071-1082. doi:10.2514/1.G000263

[6] Lyu, X., Gu, H., Wang, Y., Li, Z., Shen, S., and Zhang, F., "Design and Implementation of a Quadrotor Tail-Sitter VTOL UAV," Proceedings of the 2017 IEEE International Conference on Robotics and Automation, IEEE Publ., Piscataway, NJ, 2017, pp. 3924-3930. doi:10.1109/ICRA.2017.7989452

[7] Smeur, E. J. J., Chu, Q. P., De Croon, G. C. H. E., Remes, B., De Wagter, C., and Van der Horst, E., "Modelling of a Hybrid UAV Using Test Flight Data," Proceedings of the 2014 International Micro Air Vehicle Conference and Competition, TU Delft, Delft, The Netherlands, Aug. 2014, pp. 196-203. doi:10.4233/uuid:2eed26cf-a7e8-4a6c-ac42-89a9608e4d2a

[8] Morelli, E. A., "Dynamic Modeling from Flight Data with Unknown Time Skews," Journal of Guidance, Control, and Dynamics, Vol. 40, No. 8, 2017, pp. 2084-2092. doi:10.2514/1.G002008

[9] Hale, L. E., Patil, M., and Roy, C. J., "Aerodynamic Parameter Identification and Uncertainty Quantification for Small Unmanned Aircraft," Journal of Guidance, Control, and Dynamics, Vol. 40, No. 3 , 2017, pp. 680-691. doi:10.2514/1.G000582

[10] Uhlig, D. V., and Selig, M. S., "Modeling Micro Air Vehicle Aerodynamics in Unsteady High Angle-of-Attack Flight," Journal of Aircraft, Vol. 54, No. 3, 2017, pp. 1064-1075. doi: $10.2514 / 1 . C 033755$ 
[11] Harikumar, K., Dhall, S., and Bhat, M. S., "Nonlinear Modeling and Control of Coupled Dynamics of a Fixed Wing Micro Air Vehicle," Proceedings of the 2016 IEEE Indian Control Conference, IEEE Publ., Piscataway, NJ, 2016, pp. 318-323. doi:10.1109/INDIANCC.2016.7441153

[12] Tsiotras, P., and Mesbahi, M., "Toward an Algorithmic Control Theory," Journal of Guidance, Control, and Dynamics, Vol. 40, No. 2, 2017, pp. 194-196. doi:10.2514/1.G002754

[13] Franz, R., Milam, M., and Hauser, J., "Applied Receding Horizon Control of the Caltech Ducted Fan," Proceedings of the 2002 IEEE American Control Conference, IEEE Publ., Piscataway, NJ, 2002, pp. 3735-34740. doi:10.1109/ACC.2002.1024508

[14] Moore, J., Cory, R., and Tedrake, R., "Robust Post-Stall Perching with a Simple Fixed-Wing Glider Using LQR-Trees," Bioinspiration and Biomimetics, Vol. 9, No. 2, 2014, Paper 025013. doi:10.1088/1748-3182/9/2/025013

[15] Verling, S., Stastny, T., Battig, G., Alexis, K., and Siegwart, R., "ModelBased Transition Optimization for a VTOL Tailsitter," Proceedings of the 2017 IEEE International Conference on Robotics and Automation, IEEE Publ., Piscataway, NJ, 2017, pp. 3939-3944. doi:10.1109/ICRA.2017.7989454

[16] Velasco-Carrau, J., Garcia-Nieto, S., Salcedo, J. V., and Bishop, R. H., "Multi-Objective Optimization for Wind Estimation and Aircraft Model Identification," Journal of Guidance, Control, and Dynamics, Vol. 39, No. 2, 2016, pp. 372-389. doi:10.2514/1.G001294

[17] Theys, B., Dimitriadis, G., Hendrick, P., and De Schutter, J., "Experimental and Numerical Study of Micro-Aerial-Vehicle Propeller Performance in Oblique Flow," Journal of Aircraft, Vol. 54, No. 3, 2017, pp. 1076-1084. doi:10.2514/1.C033618

[18] Schmidt, D. K., "Stability Augmentation and Active Flutter Suppression of a Flexible Flying-Wing Drone," Journal of Guidance, Control, and Dynamics, Vol. 39, No. 3, 2016, pp. 409-422. doi:10.2514/1.G001484

[19] Kang, H., Shen, J., and Kreshock, A. R., "Validation of Comprehensive Analysis for Tiltrotor Whirl Flutter Predictions," Journal of Guidance,
Control, and Dynamics, Vol. 54, No. 4, 2017, pp. 1566-1570. doi:10.2514/1.C034272

[20] Kunz, D. L., "Analysis of Proprotor Whirl Flutter: Review and Update," Journal of Aircraft, Vol. 42, No. 1, 2005, pp. 172-178. doi: $10.2514 / 1.4953$

[21] Stevens, B. L., Lewis, F. L., and Johnson, E. N., Aircraft Control and Simulation, Wiley-Interscience, New York, 2015, pp. 1-59, Chap. 1.

[22] Anderson, J. D., Fundamentals of Aerodynamics, McGraw-Hill, New York, 2010, pp. 3-388, Chaps. 1-4.

[23] Cory, R., and Tedrake, R., "Experiments in Fixed-Wing UAV Perching," AIAA Guidance, Navigation and Control Conference and Exhibit, AIAA Paper 2008-7256, 2008. doi:10.2514/6.2008-7256

[24] Frank, A., McGrew, J. S., Valenti, M., Levine, D., and How, J. P., "Hover, Transition, and Level Flight Control Design for a SinglePropeller Indoor Airplane," AIAA Guidance, Navigation and Control Conference and Exhibit, AIAA Paper 2007-6318, 2007. doi: $10.2514 / 6.2007-6318$

[25] Sobolic, F., and How, J. P., "Nonlinear Agile Control Test Bed for a Fixed Wing Aircraft in a Constrained Environment," AIAA Infotech Aerospace Conference, AIAA Paper 2009-1927, 2009. doi:10.2514/6.2009-1927

[26] Parrilo, P. A., and Lall, S., "Semidefinite Programming Relaxations and Algebraic Optimization in Control," European Journal of Control, Vol. 9, No. 3, 2003, pp. 307-321. doi:10.3166/ejc.9.307-321

[27] Higham, N., "Computing a Nearest Symmetric Positive Semidefinite Matrix," Linear Algebra and its Applications, Vol. 103, May 1988, pp. 103-118. doi:10.1016/0024-3795(88)90223-6

[28] McCormick, B. W., Aerodynamics of V/STOL Flight, Dover, New York, 1998, pp. 212-231, Chap. 8.

[29] Leishman, J. G., Principles of Helicopter Aerodynamics, Cambridge Univ. Press, New York, 2006, pp. 81-90, Chap. 2.

[30] Itasse, M., Moschetta, J. M., Ameho, Y., and Carr, R., "Equilibrium Transition Study for a Hybrid MAV," International Journal of Micro Air Vehicles, Vol. 3, No. 4, 2011, pp. 229-245. doi:10.1260/1756-8293.3.4.229 